\title{
Taxonomic studies of lycophytes and ferns from the Pan-Himalaya (II): Crepidomanes, Didymoglossum and Vandenboschia (Hymenophyllaceae)
}

\author{
T. Y. Nwe ${ }^{1,2}$, A. P. Shalimov ${ }^{1,3}$, X.-C. Zhang ${ }^{1}$ \\ ${ }^{1}$ State Key Laboratory of Systematic and Evolutionary Botany, Institute of Botany, Chinese Academy of Sciences (CAS), Beijing \\ 100093, China.E-mail: zhangxc@ibcas.ac.cn \\ ${ }^{2}$ Myanmar Floriculturist Association, Yangon 11201, Myanmar.E-mail: thetyunwe@gmail.com \\ ${ }^{3}$ University of Chinese Academy of Science, Beijing, 100049, China.E-mail: shalimov_ap@ibcas.cn
}

Key words: Crepidomanes, Didymoglossum, Hymenophyllaceae, morphology, Pan-Himalaya, taxonomy, Vandenboschia.

Summary. The ferns family Hymenophyllaceae is one of the most interesting basal clades of ferns, which is widely distributed in tropical and subtropical regions, less common in temperate latitudes of both hemispheres. This work deals with the taxonomic study of the representatives of the subfamily Trichomanoideae, which according to the classification of PPG 1 (2016) in the region are represented only by three genera. Following the style of the treatment of the genus Hymenophyllum, this paper is the second report of our studies of the filmy ferns from the Pan-Himalaya which is a part of the ongoing "Flora of Pan-Himalaya" project. In the present account, the taxonomy of three genera of Hymenophyllaceae: Crepidomanes, Didymoglossum, and Vandenboschia from the Pan-Himalaya are carefully revised based on morphological characters and recent works in the region. Herbarium materials kept in the National Herbarium (PE) have been fully studied and compared with collections from the Himalayas kept in European herbaria (K, BM, E). The generic and infrageneric classifications follow those of Ebihara et al. (2006) which recognized only nine genera. In the Himalayas, only four genera represented, i. e., Hymenophyllum (10 species), Crepidomanes (5 species), Didymoglossum (1 species), and Vandenboschia (2 species). The synopsis includes a key for species identification, standard taxonomic citations for the genus, sections, species. For each taxon as well as of each synonym are verified and indicated, necessary literature citation, morphology descriprion and citation of the reprehensive specimens from the Pan-Himalaya are recorded, overall distribution is mentioned and distribution in the Flora of PanHimalaya area is mapped. Although this is only a precursor of the ongoing "Flora of Pan-Himalaya" project, it is useful for identification of new collections from the area, and provides basic information for the conservation and ecological studies of the filmy ferns from the Himalayas, a very sensitive area to global changes. Crepidomanes parvifolium and Didymoglossum sublimbatum in this region are critical endangered concerning their small populations and limited distribution.

\section{Таксономические исследования плауновидных и папоротников из Гималаев и прилегающих регионов (II): Crepidomanes, Didymoglossum и Vandenboschia (Hymenophyllaceae)}

\author{
Т. Ю. Нве ${ }^{1,2}$, А. П. Шалимов ${ }^{1,3}$, С.-Ч. Жан ${ }^{1}$
}

\footnotetext{
${ }^{1}$ Государственная ведущая лаборатория систематики и эволющионной ботаники, Институт ботаники, Китайская Академия наук (КАН), Пекин 100093, Китай. E-mail: zhangxc@ibcas.ac.cn

${ }^{2}$ Acсоциация иветоводов Мьянмы, Янгон 11201, Мьянма. E-mail: thetyunwe@gmail.com

${ }^{3}$ Университет Академии наук Китая, Пекин, 100049, Kuтай.E-mail: shalimov_ap@ibcas.cn
} 
Ключевые слова: морфология, Гималаи, таксономия, Crepidomanes, Didymoglossum, Hymenophyllaceae, Vandenboschia.

Аннотация. Папоротники семейства Нуmenophyllaceae являются одной из интереснейших базальных клад, представители которой широко распространены в тропических и субтропических регионах, реже в умеренных широтах обоих полушарий. Данная работа посвящена таксономическому исследованию видов подсемейства Trichomanoideae, которое по классификации авторов PPG 1 (2016) в данном регионе представлено только тремя родами. Вслед за обработкой рода Hymenophyllum, эта статья является продолжением наших исследований пленчатолистных папоротников из Гималаев и прилегающих регионов, и является частью продолжающегося проекта "Flora of Pan-Himalaya". В настоящей работе представлена таксономическая обработка для пангималайского региона трех родов из семейства Hymenophyllaceae: Crepidomanes, Didymoglossum и Vandenboschia, которые были тщательно пересмотрены с учетом последних работ по данной территории. Изучены гербарные материалы, хранящиеся в гербариях Китая (РЕ) и Европы (K, ВМ, Е). Родовая и подродовая классификация приводятся согласно работе Ebihara et al. (2006), где авторы признают только девять родов. В Гималаях представлены четыре рода семейства: Hymenophyllum (10 видов), Crepidomanes (5 видов), Didymoglossum (1 вид) и Vandenboschia (2 вида). Конспект включает ключ для определения видов, стандартное таксономическое цитирование для родов, секций и видов. Для каждого вида указываются синонимы, информация о типе и месте его хранения (также для синонимов), цитируется основная литература, приводятся подробное морфологическое описание и изученные образцы из пангималайского региона, указывается распространение в пределах области «Флоры» и общее распространение. Представленная работа полезна как отправная точка для выявления новых находок представителей Нуmenophyllaceae в этом районе и как основа для планирования мероприятий по сохранению пленчатолистных папоротников Гималаев, которые очень чувствительны к глобальным изменениям климата. Crepidomanes parvifolium и Didymoglossum sublimbatum в этом регионе находятся под угрозой исчезновения вследствие небольшой численности популяций и ограниченного распространения.

The filmy fern family Hymenophyllaceae is one of the largest families among the modern representatives of pteridophytes, comprising ca. 600 species (Iwatsuki, 1990) or 434 species (PPG I, 2016). The peculiarities in morphological characters make them distinct from other ferns. The plant body is transparent and thin sheet-like structure. Terminal sori occur at the end of veins. The number of spores in each sporangium ranges from 64 to 512 . Often, the spores begin to germinate in the sporangium, and they have the ability to germinate rapidly. Filmy species occur in very humid conditions in the tropical, subtropical and temperate regions of both Old and New Worlds, with highest diversity in mountainous tropical rain forests.

In the present study, a comprehensive review of the relevant literatures and a thorough examination of herbarium specimens from the Pan-Himalaya deposited at the Chinese National Herbarium (PE) and other European herbaria (BM, E, K) are conducted. The Pan-Himalaya comprises the northeastern Afghanistan, northern Pakistan, northern India, Nepal, Bhutan, northern Myanmar, and southwest China (S Tibet, SE Qinghai, SE Gansu, W Sichuan, and NW Yunnan). Modern systematics of this group is often disputed. Traditionally, only two genera were recognized in the family Hymenophyllaceae: Hymenopyllum and Trichomanes. This scheme was expanded by
Morton (1968), who hierarchically placed many subgenera, sections and subsections under these two genera. Conversely, Copeland (1947) split this family into 34 genera. Recently, a new classification of this family was proposed by Ebihara et al. (2006), based on the past as well as recent investigation involving molecular phylogenetic analyses. The classification of Ebihara et al. (2006) divided the family into nine genera, of which four genera, i. e., Crepidomanes, Didymoglossum, Vandenboschia, and Hymenophyllum are represented in the PanHimalaya.

Hymenophyllaceae Link, 1833, Handb. Erken. Gew. 3: 36 as "Hymenophyllaceae"; C. Presl, 1843, Hymenophyllaceae 1-93; H. S. Kung, 1988, Fl. Sichuan. 6: 138; K. Iwats. 1990, in Kramer et Green, Fam. Gen. Vasc. Pl. 1: 157; Z. R. He, 2006, Fl. Yunnan. 20: 168.

Plants epiphytic, terrestrial or lithophytic, mostly small or minute to medium-sized. Rhizome long to short-creeping, sub-erect or erect, usually slender, often covered with hairs when young, without scale, simple or sometimes irregularly branching, root present or if absent root-like shoot present. Stipe winged or wingless. Lamina varying from simple to pinnately decompound or flabellate, digitate, dichotomous, or even irregularly divided, usually one cell thick except for the vein and in some 
genera; veins usually free, rarely anastomosing, false-veins (thickenings resembling veins but lacking any vascular tissues); without stomata. Sori terminal on veins, solitary, at the apex of pinnules or short lateral lobes, or marginal on simple to pinnatifid fronds; involucres cup-shaped to bivalvate, tubular or obconic with the lips often widely spreading; receptacles formed by extension of the vein, short, capitates or clavate, or long and included or projecting; sporangia clustered along the receptacle, short stalked to sub-sessile, maturing basipetally; annulus complete, oblique or almost transverse; dehiscence irregularly; spores globosetrilete, tetrahedral, without perispore. Chromosome number $x=8,11,12,13,17,21,22,26,28,36,54$ (base numbers $x=8,54$ are doubtful).

\section{Key to genera of Hymenophyllaceae from the Pan-Himalaya}

1. Root-like shoot absent 2

+ Root-like shoots present 3

2. Rhizomes usually filiform, nearly glabrous; involucres bivalvate, lips entir or serrate, receptacle included 1. Hymenophyllum

+ Rhizomes rather thick, covered with brown to bright brown multicellular hairs; involucres not bivalvate, lip sometimes dilate, receptacle longexserted 4. Vandenboschia

3. Blade usually simple, or sometimes lobed to pinnatifid; venation catadromous, false veinlet present or absent, if present false veinlet parallel not connected to the true veins ....... 2. Didymoglossum

+ Blade simple to quadripinnatifid; venation anadromous, often flabelliform, false veinlet longitudinal, parallel to the true veins present

3. Crepidomanes

\section{HYMENOPHYLLUM}

For the taxonomic treatment of the genus Humenophyllum, see Nwe and Zhang (2017, Turczaninowia, 20, 2: 75-96).

\section{DIDYMOGLOSSUM}

Didymoglossum Desv., 1827, Mém. Soc. Linn. Paris 6: 330. $\equiv$ Trichomanes sect. Didymoglossum (Desv.) T. Moore, 1857, Index Fil.: CX. $\equiv$ Trichomanes subg. Didymoglossum (Desv.) C. Chr., 1857, Index Filic.: XIV.

Lectotype: Didymoglossum muscoides (Sw.) Desv. (= Didymoglossum hymenoides (Hedw.) Copel.), chosen by Christensen (1906), as 'Trichomanes hymenoides Hedw.'.

= Hemiphlebium sect. Lecanium Prantl, 1875, Untersuch. Morph Gefasskrypt. 1: 46, based on
Lecanium C. Presl, 1843, Hymenophyllaceae 11, t. 1. nom. illeg.; non Reinw., 1825, Flora 8(2, Beil.): 48. $\equiv$ Trichomanes sect. Lecanium (Prantl) Christ, 1897, Farnkr. Erde 25. 三 Lecanolepis Pic. Serm., 1973, Webbia 28(2): 449.

Type: Lecanium membranaceum (L.) C. Pres1 ( $\equiv$ Didymoglossum membranaceum (L.) Vareschi).

= Didymoglossum sect. Flabellate C. Presl, 1843, Hymenophyllaceae 23.

Lectotype: Didymoglossum sphenoides (Kunze) C. Presl (= Didymoglossum punctatum (Poir.). Desv.), chosen by Morton (1968).

$=$ Didymoglossum sect. Pinnata C. Pres1, 1843, Hymenophyllaceae 23.

Lectotype: Didymoglossum muscoides (Sw.) Desv. (= Didymoglossum hymenoides (Hedw.) Copel.), chosen by Morton (1968).

$=$ Hemiphlebium C. Presl, 1843, Hymenophyllaceae 25, t. IX. $\equiv$ Trichomanes sect. Hemiphlebium (C. Presl) T. Moore, 1857, Ind. Fil.: CX. $\equiv$ Trichomanes subg. Hemiphlebium (C. Presl) Christ, 1897, Farnkr. Erde 23.

Type: Hemiphlebium pusillum (Sw.) C. Presl (三 Didymoglossum pusilum (Sw.) Desv.).

Plant epilithic or low-epiphytic. Rhizomes longcreeping, filiform, frequently branching, densely covered with dark hairs, root absent, root-like shoot present. Lamina small, usually simple, or sometimes lobed to pinnatifid, elliptic to narrowly ovate, often reduced the stipe; venation catadromous, often flabelliform, submarginal false veinlets absent, longitudinal false veinlets parallel to the true veins present, cell walls thin and straight. Sori epitactic or pantotactic, often immersed in the laminae, campanulate, lips bilabiate or occasionally truncate, receptacle exserted.

One species was found in Pan-Himalaya, more than 20 species are distributed throughout the tropics, mainly in the New World.

Didymoglossum sublimbatum (Müll. Berol.) Ebihara et K. Iwats., 2006, Blumea 51: 236. 三 Trichomanes sublimbatum Müll. Berol., 1854, Bot. Zeitung (Berlin) 12: 737; Copel., 1933, Phil. J. Sci. 51: 198. pl. 28.f. 1-2; Tardieu et C. Chr., 1939, Fl. Gén. I.-C. 7(2): 62; Holttum, 1954, Rev. Fl. Mal. 2: 92. f. 29. $\equiv$ Microgonium sublimbatum (Müll. Berol.) Bosch, 1861, Hymen. Jav. 6, t. 2; Tagawa et K. Iwats., 1979, Fl. Thail. 3: 94; Ghosh et al., 2004, Pterid. Fl. E. India 1: 249; Panigrahi et Sarn. Singh, 2005, Ferns Fern-Allies Arunachal Pradesh 1: 353; Z. R. He, 2006, Fl. Yunnan. 20: 190. 
Type: Indonesia. "Partia, Java, Zollinger, 1899, 3500" (BM, BO, L, GH [GH00022271], [US00134626]); “865” (BM, BO, K [K000375036], L) (syntypes).

Plants $1-2.8 \mathrm{~cm}$ tall. Rhizome long creeping, ca. $0.2 \mathrm{~mm}$ in diam., densely hairy; hairs brown, unicellular, oblong-linear, twisted, less than 0.4 $\mathrm{mm}$ long, root like shoot present. Stipes 5-9 mm apart, 1-3 mm long, densely hairy like the rhizome, narrowly winged except the base. Lamina simple, oblong to linear-oblong, $1-2.5 \mathrm{~cm} \times 3.5-7 \mathrm{~mm}$, base subtruncate to cuneate, gradually narrowing downwards, margin repand to sinuated lobed, lobe a quarter ways to costae, apex round or obtuse. Main vein bearing only sori; sometimes lateral vein present; lateral veins 3-4 paris, simple or forked, marginal false veinlet absent; false vein present, obliquely parallel to the lateral veins, long, ending within the margin, several lines up to 15 between adjacent veins. Sori 1-5 on apical part of frond, usually terminal on lobes; involucres tubular, 1.5$2 \times 0.7-1 \mathrm{~mm}$, completely immersed in frond, lips flaring and dilated, mouth up to $2 \mathrm{~mm}$ in diam., receptacle exerted. See Fig. $1 \&$ 9A.

Note: Formerly, this species was placed in the genus Microgonium (Copeland, 1938; 1947). But in the new classification system of Ebihara et al. (2006), it was placed into the genus Didymoglossum because of the lack of submarginal false veinlets.

Distribution in Pan-Himalaya: China (Yunnan, Xizang), Bhutan.

General distribution: China (Taiwan, Guangxi, Guizhou, Yunnan, Xizang), India (Khasia, Changlang, Assam), Myanmar, Thailand, Vietnam, Malaysia, Indonesia (Java, New Guinea), Papua New Guinea. This species is vulnerable in India by C. R. Fraser-Jenkins (2012).

Habitat: On muddy rocks or rarely on basal tree-trunks in dense forests, at $850 \mathrm{~m}$.

Representative specimen examined: CHINA, YUNNAN, Xishuangbanna, $750 \mathrm{~m}, \mathrm{~K}$. H. Shing et al. 6994 (PE). XIZANG, Southeast Xizang, Medog, epiphytic on rocks by riverbank, alt. $850 \mathrm{~m}, \mathrm{M}$. Z. Wang 800808 (PE).

Note: $\mathrm{Wu}$ et al. (2016) recorded Didymoglossum sublimbatum as a new species to the flora of Xizang.

\section{CREPIDOMANES}

Crepidomanes (C. Presl) C. Presl, 1849, Epimel. Bot.: 258. $\equiv$ Trichomanes subg. Crepidomanes C. Presl, 1849, Epimel. Bot.: 17. 三 Trichomanes sect. Crepidomanes (C. Presl) Prantl, 1875, Untersuch, Morph. Gefasskrypt. 1: 51.
Type: Trichomanes intramarginale Hook. et Grev. (三 Crepidomanes intramarginale (Hook. et Grev.) C. Presl).

Five species were found in Pan-Himalaya and more than 30 species are distributed throughout the Old World tropics to northern temperate regions.

\section{Key to the sections}

1. Laminae elliptic to subdeltoid; false veinlets present; sori tubular not immersed in the laminae .. 1. Crepidomanes

+ Laminae flabellate to narrowly ovate, false veinlets absent; sori campanulate often immersed in the lamina

2. Gonocormus

\section{Section Crepidomanes}

Trichomanes subg. Minora Prantl, 1875, Untersuch. Morph. Gefasskrypt. 1: 51.

Lectotype: Trichomanes intramarginale Hook. et Grev. (三 Crepidomanes intramarginale (Hook. et Grev.) Copel.), chosen by Morton (1968).

$=$ Trichomanes sect. Taschneria C. Presl ex C. Chr., 1906, Ind. Fil. XV [Taschneria C. Presl, 1849, Epim. Bot. 258, nom. nud.] $\equiv$ Crepidomanes sect. Taschneria (C. Presl ex C. Chr.) K. Iwats., 1984, Acta Phytotax. Geobot. 35(4-6): 175.

Type: Trichomanes filicula Bory ex Willd. (= Crepidomanes bipunctatum (Poir.) Copel.).

Plants epiphytic or rock, rarely terrestrial. Rhizomes long-creeping, frequently branching, filiform, densely covered with dark hairs, root absent, root-like shoot present. Lamina simple to quadripinnatifid, elliptic to subdeltate some dwarf species digitately divided. Venation anadromous, false veinlets mostly present but some species absent, submarginally (continuous or interrupted) and/or parallel (but not connected) to true veins, cell wall thin and straight. Sori paratactic, tubular, campanulate or obconic, often broadly winged, lips usually bilabiate, sometimes entire or dilated, receptacle exserted.

Five species were found in Pan-Himalaya and more than 20 species are distributed throughout the Paleotropics.

\section{Key to the species}

1. False veinlet present 2

+ False veinlet absent; frond bipinnatifid, oblongovate ..................................... 4. C. schmidtianum

2. Fronds small, not more than $1 \mathrm{~cm}$, lamina simple, elliptic, obcordate to oval 3. C. parvifolium 
+ Fronds more than $1 \mathrm{~cm}$ long, lamina not simple

3. Lamina 2 or 3-pinnate, oblong or narrowly to broadly ovate; stipe winged with blackish hairs; submarginal false veinlet present

1. C. bipunctatum

+ Lamina 2-pinnate to 3-pinnatifid, broadly ovate to lanceolate; stipe winged without hairs submarginal false veinlet absent

2. C. latealatum

1. Crepidomanes bipunctatum (Poir.) Copel., 1938, Phil. J. Sci. 67: 59; Ching, 1959, Fl. Reip. Pop. Sin. 2: 162. pl. 12. f. 8; Seriz., 1975, Sci. Rep.
Takao Mus. N. H. 7: 15; Tagawa et K. Iwats., 1979, Fl. Tahil. 3: 90; Ghosh et al., 2004, Pterid. Fl. E. India 1: 251. 三 Trichomanes bipunctatum Poir., 1808, Enc. 8: 69; Bedd., 1868, Ferns Brit. Ind. pl. 283; Copel., 1933, Phil. J. Sci. 51: 177. pl. 18. f. 1-4; Tard. et C. Chr., 1939, Fl. Gén. I.-C. 7(2): 63; Holttum, 1954, Rev. Fl. Mal. 2: 99. f. 35.

Type: Madagascar. "Cette plante a ete decouverte a l'ile de Madagascar, par M. Aubert du PetitThouars s.n." - P [P00482602] (holotype).

$=T$. bilabiatum Nees et Blume, 1823, Nova Acta 11: 123, t. 2; Copel., 1933, Phil. J. Sci. 51: 179. pl. 18. f. 5-6; Holttum, 1954, Rev. Fl. Mal. 2: 99. f. 36; Sledge, 1968, J. Linn. Soc. Bot. 60: 306. $\equiv$

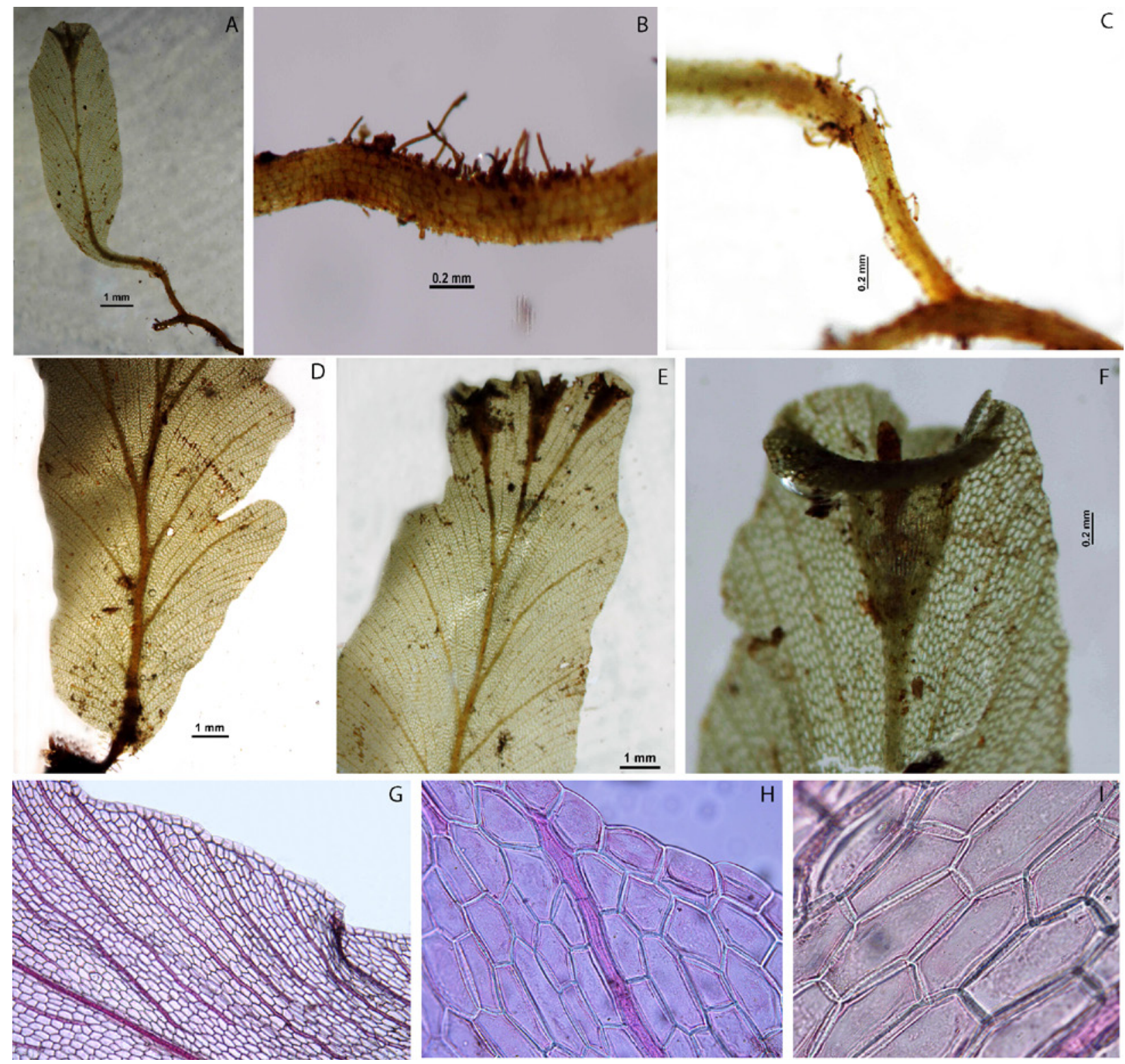

Fig. 1. Didymoglossum sublimbatum (Müller Berol.) Ebihara et K. Iwats. A. Frond, B. Rhizome, C. Stipe, D. Part of frond with sinus lobed, E. Fertile frond, F. Sorus, G. False veinlet longditudinal parallel to the main vein, H. Marginal cells, I. Lamina cells. A-I: K. H. Shing et al. 6994 (PE 02050370). 
Crepidomanes bilabiatum (Nees et Blume) Copel., 1938, Phil. J. Sci. 67: 59; K. Iwats., 1958, Acta Phytotax. Geobot. 17(6): 161; Dixit, 1984, Census Ind. Pterid.: 91; Panigrahi et Sarn. Singh, 2005, Ferns Fern-Allies Arunachal Pradesh 1: 333.

Type: Indonesia. "Java, Jawa Barat, Bantam" L [L0544641] (holotype).

$=C$. dilatatum Ching et Ch. H. Wang, 1959, Acta Phytotax.Sin. 8: 137, t. 19. f. 13; Ching, 1959, Fl. Reip. Pop. Sin. 2: 164.

Type: China. "Hainan: Tsai Hsien, Sha-pao Shan, W. T. Tsang 598, growing on the side of ravine, 30 May 1928" - PE [PE-00042624!] (holotype).

= Didymoglossum griffithii Bosch, 1863, Ned. Kruid, Arhc. 5(2): 141; Copel., 1933, Phil. J. Sci. 51: 193. $\equiv$ Crepidomanes griffithii (Bosch) Dixit et Grosh, 1984, Census Ind. Pterid.: 91.

Type: Myanmar. "Mergui, Griffith s.n." - K [K001090181] (holotype).

Plants $1.5-11 \mathrm{~cm}$ tall. Rhizome long creeping, wiry, $0.2-0.7 \mathrm{~mm}$ in diam., irregularly branching, densely covered with reddish brown or dark brown short hairs, unicellular, root like shoot present. Stipes remote, $0.4-4 \mathrm{~cm}$ apart, $0.2-3.5 \mathrm{~cm} \times 0.4-1.8$ $\mathrm{mm}$, narrowly winged throughout or up to middle; wings ciliate on half portion of the stipe, blackish, unicellular, discontinuous false veinlet present. Rachis and costae winged throughout, glabrous or abaxial surface with club-shaped hairs. Lamina 2 or 3-pinnate, oblong or narrowly to broadly ovate, $1.5-9 \times 1-4 \mathrm{~cm}$, glabrous; pinnae 4-6 pairs, closely spaced, alternate, subsessile, ovate to oblong, 1-1.6× $0.5-1 \mathrm{~cm}$; pinnules 3 or 4 pairs, closely spaced, alternate, sessile, obovate to broadly cuneate, base unequally cuneate, apex subtruncate; ultimate segments closely spaced, narrowly linear, 2-4 $\times$ $0.4-0.8 \mathrm{~mm}$, margin entire or slightly crisped, apex round, obtuse or acute. Lamina cell irregular in shaped, cell walls thin and straight. Veins dichotomous, free, stout, slightly raised on each surface, club shaped hairy, reddish; submarginal false veinlets continuous, 1 or 2 rows of cell present between false veinlets and margin of lamina, other striae few, short, 1 row parallel through the main vein. Sori apical on acroscopic segments, numerous, $1-5$ per each pinna; involucres narrowly elliptic, $2-2.3 \times 0.7-1.3 \mathrm{~mm}$, winged, lips bilabiate, triangular at tips; receptacles exserted, dark brown, 2-3 mm. See Fig. 2 \& 9B.

Chromosome number: $2 n=72$ (Mitui, 1975).

Distribution in Pan-Himalaya: India (Sikkim, Kameng).
General distribution: Africa, Madagascar, China (Guangdong, Guangxi, Guizhou, Hunan, Sichuan, Taiwan, Yunnan), Japan, India (Sikkim, Kameng, West Bengal, Meghalaya, Manipur, Mizoram, Nagaland, western part of India), Myanmar, Thailand, Malaysia, Indonesia, The Philippines, Australia (Queensland), Pacific Islands (Melanesia, Micronesia, Polynesia).

Habitat: On mossy tree-trunks or on mossy rocks in dense forests at 300-2000 m.

Representative specimen examined: Although it is a widespread species in Asia, it is scarce in the Himalayas. Most collections in PE are out of the Pan-Himalaya flora. The illustrations are from the collection from South Yunnan: Pingbian, $1500 \mathrm{~m}$, W. M. Zhu 111 (PE).

2. Crepidomanes latealatum (Bosch) Copel., 1938, Phil. J. Sci. 67: 60; H. Ito, 1944, Fil. Jap. Ill. pl. 475; Ching, 1959, Fl. Reip. Pop. 2: 165; K. Iwats., 1975, Fl. E. Himalaya 8: 171; Tagawa et K. Iwats., 1979, Fl. Thail. 3: 89; Dixit, 1984, Census Ind. Pterid.: 91; H. S. Kung, 1988, Fl. Sichuan. 6: 154; K. Iwats., 1988, Bull. Univ. Mus. Univ. Tokyo 31: 252; Ghosh et al., 2004, Pterid. Fl. E. India 1: 252; Z. R. He, 2006, Fl. Yunnan. 20: 196. 三 Didymoglossum latealatum Bosch, 1863, Ned. Kruidk. Arch. 5(2): 138; Copel., 1933, Phil. J. Sci. 51: 192, pl. 25, 26. $\equiv$ Trichomanes latealatum (Bosch) Christ, 1896, Verh. Nat. Ges. Basel XI; Tard. et C. Chr., 1939, Fl. Gén. I-C. 7(2): 64; Holt., 1954, Rev. Fl. Mal. 2: 101.

Type: India. "India orientalis, Assam, Griffith s.n." - K [K000235197] (holotype).

= Didymoglossum racemulosum Bosch, 1863, Ned. Kruid. Arch. 5(2): 137; Copel., 1933, Phil. J. Sci. 51: 193; Z. R. He, 2006, Fl. Yunnan. 20: 193. $\equiv$ Crepidomanes racemulosum (Bosch) Ching, 1959, Fl. Pop. Sin. 2: 170; Dixit, 1984, Census Ind. Pterid.: 92.

Type: India. "India orientalis, Assam, Griffith s.n." (K).

$=$ Trichomanes acutilobum Ching, 1934, Ind. Fil. Suppl. 2: 187; Tard. et C. Chr., 1939, Fl. Gén. I-C. 7(2): 64 .

= Didymoglossum plicatum Bosch, 1863, Ned. Kruid. Arch. 5(3): 139; Copel., 1933, Phil. J. Sci. 51: 193. $\equiv$ Trichomanes plicatum (Bosch) Bedd., 1868, Ferns Br. Ind. pl. 285; Tard. et C. Chr., 1939, Fl. Gén. I-C. 7(2): 64; Sledge, 1968, J. Linn. Soc. Bot. 60: 305. $\equiv$ Crepidomanes plicatum (Bosch) Ching, 1959, Fl. Reipubl. Popularis Sin. 2: 171; Ghosh et al., 2004, Pterid. Fl. E. India 1: 252; Z. R. He, 2006, Fl. Yunnan. 20: 194. 
Lectotype: Malaysia. "Malacca, Malaya, Griffith s.n." - K (Morton, 1967, photograph 19028) [K001090208], chosen by Morton (1968).

= Didymoglossum euphlebium Bosch, 1863, Ned. Kruid. Arch. 5(2): 142; Copel., 1933, Phil. J. Sci. 51: 193. $\equiv$ Crepidomanes euphlebium (Bosch) Dixit et Grosh, 1984, Census Ind. Pterid.: 91.
Holotype: India. "India orientalis, Assam, Griffith s.n." - (K).

= Didymoglossum insigne Bosch, 1863, Ned. Kruid. Arch. 5(2): 143; Copel., 1933, Phil. J. Sci.51: 193. $\equiv$ Trichomanes insigne (Bosch) Bedd., 1868, Ferns Br. Ind. pl. 284 C; Tard. et C. Chr., 1939, Fl. Gén.I-C. 7(2): 64; Z. R. He, 2006, Fl. Yunnan. 20:
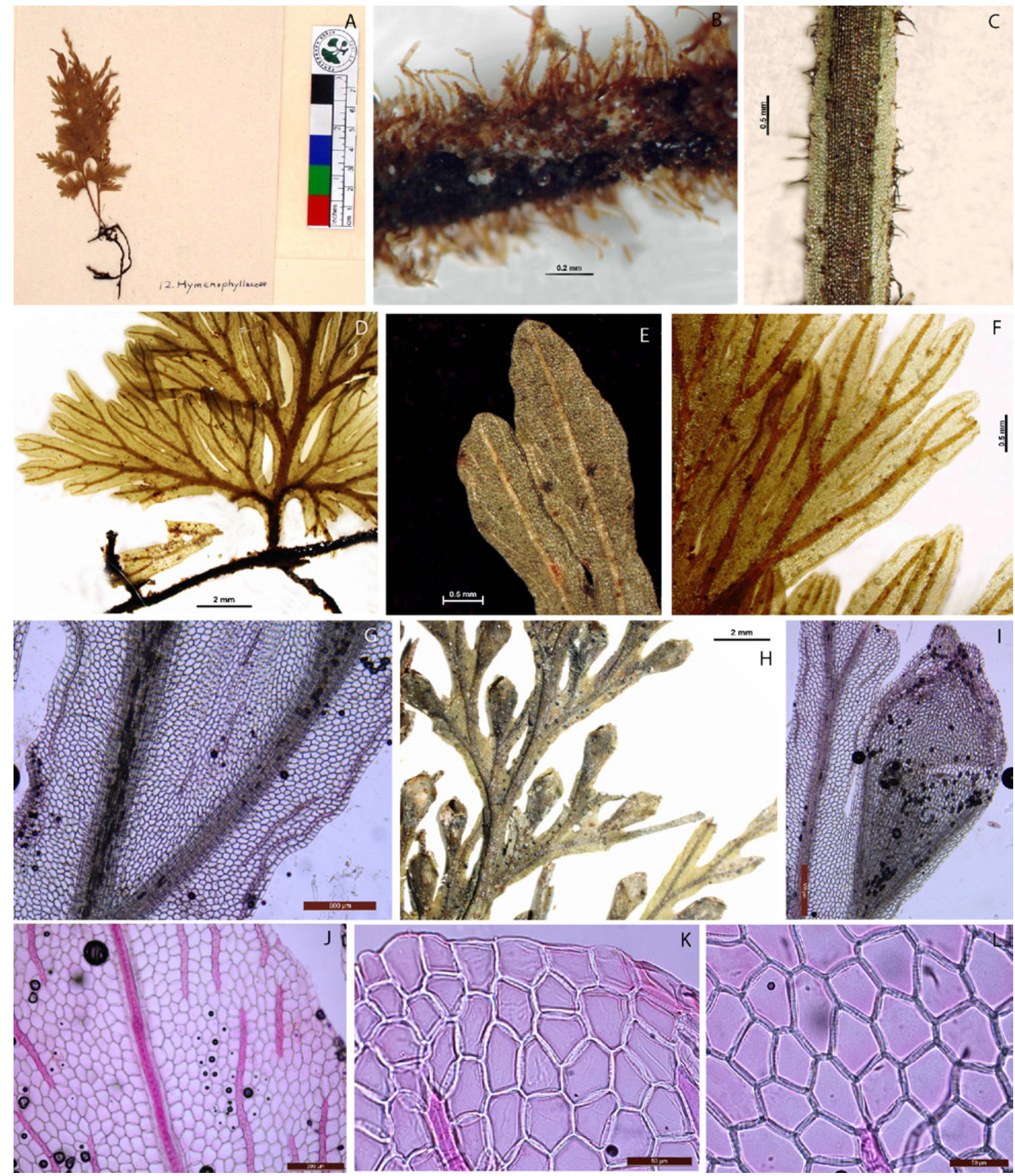

Fig. 2. Crepidomanes bipunctatum (Poir.) Copel. A. Frond, B. Rhizome, C. Stipe wing with hairs, D. Part of frond, E. Ultimate segments, F. Pinnae, G. submarginal false veinlet, H. Fertile frond, I. Sorus, J. False veinlet obliquely parallel to the main vein, K. Marginal cells, L. Lamina cells. A-L: W. M. Chu 111 (PE 01267344). 
192. $\equiv$ Crepidomanes insigne (Bosch) Fu, 1957, Ill. Handb. Chin. Pl. Pterid. 39; Ching, 1959, Fl. Reip. Pop. Sin. 2: 168. pl. 11, f. 9-12; Nakaike, 1975, Enum. Pterid. Jap. 17; Serizawa, 1975, Sci, Rep. Takao Mus. N. H. 7: 15; Iwatsuki, 1975, Fl. E. Himalaya 8: 171; Dixit, 1984, Census Ind. Pterid.: 91; Panigrahi et Sarn. Singh, 2005, Ferns Fern-Allies Arunachal Pradesh 1: 335.

Type: India. "India orientalis, Mishmee, Griffith s.n.”-K [K001090194] (holotype); [BM001019588] (isotype).

= Trichomanes viridans Mett.ex Kuhn, 1868, Linnaea 35: 389.

Type: Myanmar. "Moulmein, Parish 184 (1862)" - K.

= Trichomanes makinoi C. Chr., 1906, Ind. Fil. 644, based on Trichomanes acutum Makino ex Christ, 1896, Bull. Herb. Boiss. 4: 665; non Presl, 1843; Copel., 1933, Phil. J. Sci. 51: 195. pl. 27. $\equiv$ Crepidomanes makinoi (C. Chr.) Copel., 1938, Phil. J. Sci. 67: 105; H. Ito, 1944, Fil. Jap.Ill. pl. 476; K. Iwats., 1958, Acta Phytotax. Geobot.17(4): 71; Tagawa, 1959, Col. Ill. Jap. Pterid. 44, 192. f. 66a; H. S. Kung, 1988, Fl. Sichuan. 6: 154.

Type: Japan. "Japan, Tosa, Nov. 1887, T. Makino s.n.” - P [P00623409] (holotype); TI (isotype).

= Trichomanes tosae Christ ex Matsum., 1910, Bot. Mag. Tokyo 24: 240. $\equiv$ Crepidomanes makinoi var. tosae (Christ ex Matsum.) K. Iwats., 1958, Acta Phytotax. Geobot. 17(4): 72; Tagawa, 1959, Col. Ill. Jap. Pterid. 44, 192. f. 66b.

Type: Japan. "Tosa, Shikoku Island, Matsumura J., n. 174" - TI (holotype); P [P00623413], PE (isotype).

$=$ Crepidomanes smithiae Ching, 1959, Acta Phytotax. Sin. 8: 138; Ching, 1959, Fl. Reip. Pop. Sin. 2: 169; Z. R. He, 2006, Fl. Yunnan. 20: 192.

Type: China. "Hainan, Ng Chi Shan, Eryl Smith 1402, I, 1923" - PE [PE 00042628!] (holotype).

$=C$. chiuii Ching et P. S. Chiu, 1959, Fl. Reip. Pop. Sin. 2: 167, 355.

Type: China. "Yunnan austr.-orient., Ping-pien Hsien, Ta-wei Shan, $1400 \mathrm{~m}$ alt., W. M. Chu, n. 40" - PE [PE00042623!] (isotype).

$=C$. omeiense Ching et P. S. Chiu, 1959,

Fl. Reip. Pop. Sin. 2: 167, 355; H.S. Kung, 1988, Fl. Sichuan. 6: 152.

Type: China. "Szechuan, Omei Shan, from Chiulao-tung to Se-shiang Chu, leg. S. C. Chen, n. 10039, VIII, 16, 1953" - PE [PE00001376!] (paratype).

$=$ C. paucinervium Ching, 1959, Fl. Reip. Pop. Sin. 2: 167, 355.

Type: China. "China, Sichuan, bor.-occid., H. H. Simth 2002" - PE [PE00042626!] (isotype).
$=C$. yunnanense Ching et P. S. Chiu, 1959, Fl. Reip. Pop, Sin. 2: 169, 355; Z. R. He, 2006, Fl. Yunnan. 20: 192.

Type: China. "Yunnan, austr.-orient., Hokou, Nan-chi, 200 m alt., on tree trunks, W. M. Chu 2741 (1955)" - PE [PE00042629!] (holotype).

Plants $2-14 \mathrm{~cm}$ tall. Rhizomes long creeping, filiform, ca. $0.5 \mathrm{~mm}$ in diam., densely covered with reddish to dark brown hairs, unicellular; irregularly branching; root like shoot present. Stipes remote, $0.6-4.5 \mathrm{~cm}$ apart, reddish to dark brown, $1-2.5 \mathrm{~cm} \times$ ca. $0.5 \mathrm{~mm}$, hairy at one third of the stipe, dark brown, unicellular; winged half way or throughout, short or long striate along the margin present. Rachis and costae stramineous to reddish-brown, sparsely with club shape hairs, narrowly winged throughout, slightly zig-zag. Lamina 2- to 3-pinnatifid, broadly ovate to lanceolate, membraneous, glabrous, base cuneate to subcordate, apex acuminate to obtuse; pinnae 4-9 pairs, closely spaced, alternate, sessile, broadly ovate to ovate-lanceolate, $0.4-3 \mathrm{~cm} \times$ $3.5-6 \mathrm{~mm}$, base cuneate, apex obtuse; ultimately segments closely spaced, linear to lanceolate or broadly cuneate, margin entire, somewhat crisped, apex obtuse to acuminate. Lamina cells rectangular or irregular, cell wall uniformity thin and straight, marginal row of cells rectangular or irregular. Veins dichotomous obviously raised on each surface, dark brown, light black or green, glabrous, marginal false veinlets absent, other striae variable in length, longitudinal parallel to the vein, $0.2-1 \mathrm{~mm}$ long. Sori upper part of the frond, apical on short acroscopic segment, 1-6 per pinna; involucres tubular, elliptic, or obconic, $1.5-2 \times 0.5-1 \mathrm{~mm}$, winged or wingless, mouse dilated, lips rounded, acute to triangular, false veinlets present, short, longitudinal; receptacles exserted. See Fig. 3 \& 9C.

Chromosome number: $2 n=72$ (Mitui, 1967, 1968, 1973).

Distribution in Pan-Himalaya: China (Sichuan: Boaxing; Yunnan: Yanyuan, Weixi, Gongshan; S. Xizang: Chayu, Medog, Bomi), Bhutan, W, C and E Nepal, India (Himachal Pradesh: Chamba, Dalhousie, Panjpulta; Darjeeling; Sikkim; Uttra Pradesh: Dehra Dun, Mussorie, Kameng, Papum Pare, Subansiri).

General distribution: China (Anhui, Fujian, Guangdong, Guangxi, Guizhou, Hainan, Hunan, Jiangxi, Sichuan, Taiwan, Yunnan, Zhejiang), Japan, India (Assam, south and west of India), Sri Lanka, Vietnam, Malaysia, Australia.

Habitat: On tree-trunk, mossy rocks or cliffs in forests from $1650-2800 \mathrm{~m}$ altitudes. This is the 
one of the commonest of Himalaya species and throughout the tropics of the world.

Note: This species is a widespread, variable species base on the form and size of the fronds. Many names have been proposed by many authors.

Representative specimen examined: CHINA, GANSU, Wenxian: 1100 m, D. E. Bouffort et al. 37698 (PE, C. paucinervium). 1800 m, Y. P. Xu 1625 (PE, C. paucinervium). 1300 m, Y. P. Xu 1769
(PE, Crepidomanes latealatum). XIZANG, Bomi: $2400 \mathrm{~m}, \mathrm{~T}$. S. Ying et D. Y. Hong 650538 (PE, C. plicatum). Chayu: 2000 m, Tibetan Exp. Team 73946 (PE, C. latealatum). Medog: Tibetan Exp. Team (Vegetation Group) 3138 (PE, C. racemulosum). 750 m, South Tibet Exp. Team (STET) STET2464 (PE, C. latealatum). YUNNAN, Eryuan, Qin Dynasty 24852 (PE, C. latealatum). Gongshan: $2300 \mathrm{~m}$, C. W. Wang 66813 (PE, C. plicatum). $2000 \mathrm{~m}$,

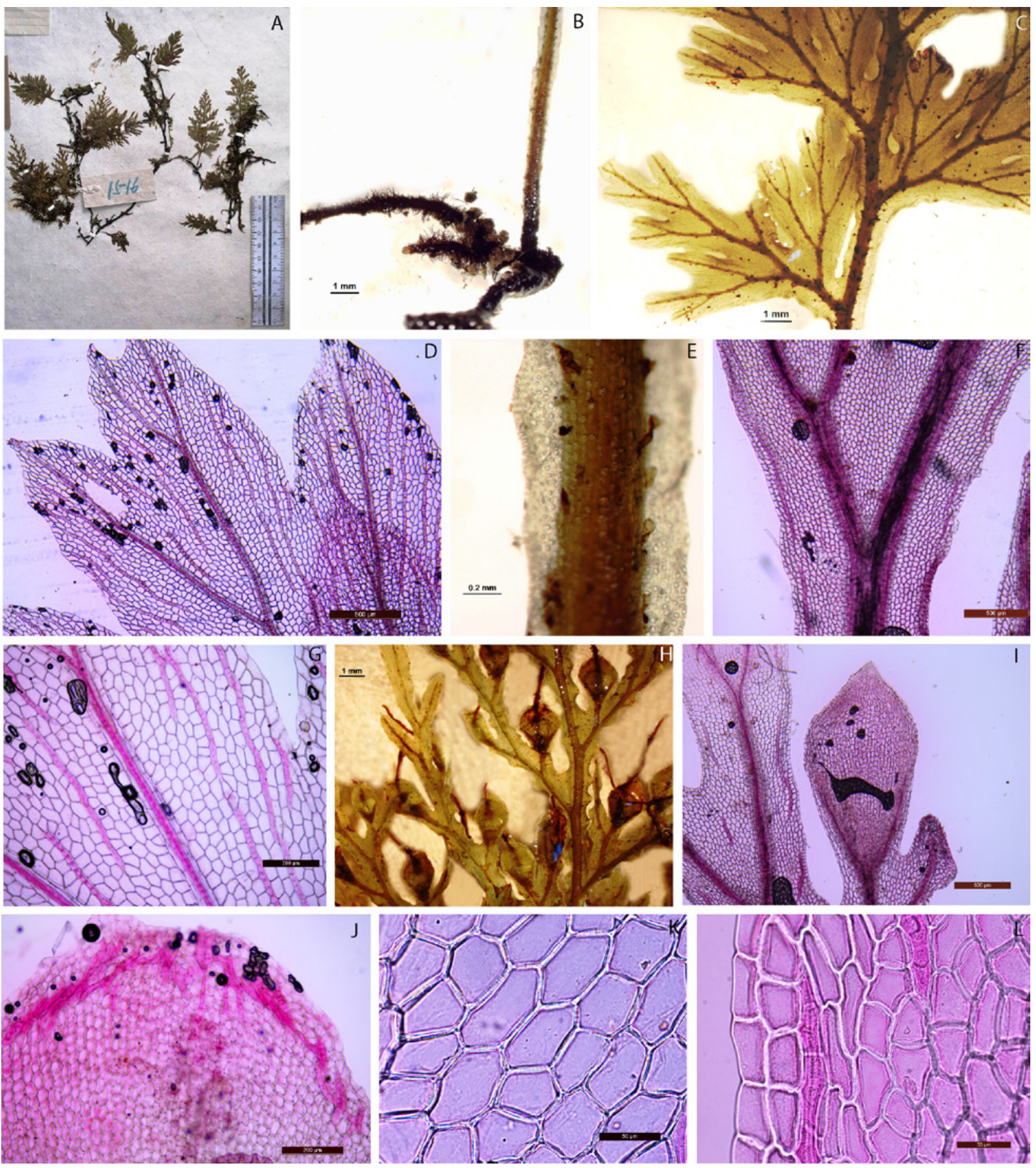

Fig. 3. Crepidomanes latealatum (Bosch) Copel. A. Frond, B. Rhizome and stipe, C. Part of frond, D. false veinlet obliquely parallel to the main vein E. Ultimate segments, F. Pinnae, G. Submarginal false veinlet, H. Fertile frond, I. Sorus, J. False veinlet obliquely parallel to the main vein, K. Marginal cells, L. Lamina cells. A-L: K. H. Shing et K. Y. Lang 1516 (PE 00239508). 
Y. C. Xu 474 (PE, C. plicatum). Weixi: $3000 \mathrm{~m}$, C. W. Wang 64570 (PE, C. lineatum). SICHUAN, Baoxing: 2500 m, K. C. Kuan et W. T. Wang 3037 (PE, C. paucinervium).

3. Crepidomanes parvifolium (Baker) K. Iwats., 1985, J. Fac. Sci. Univ. Tokyo, Sect. 3, Bot. 13: 535. $\equiv$ Hymenophyllum parvifolium Baker, 1866, J. Linn. Soc. Bot. 9: 340. pl. 8. f. E; Bedd., 1867, Ferns Br. Ind. pl. 225. $\equiv$ Trichomanes parvifollium (Baker) Copel., 1933, Phil. J. Sci. 51: 211. $\equiv$ Microgonium parvifolium (Baker) Tagawa et K. Iwats., 1975, Acta Phytotax. Geobot. 26: 169; Z. R. He, 2006, Fl. Yunnan. 20: 190.

Type: Myanmar. "Moulmein, C. Parish s.n. 1862”-K [K001090172] (holotype), E [E00413867] (isotype).

$=$ M. multifolium Tagawa et K. Iwats., 1967, Acta Phytotax. Geobot. 22: 98. f. 2.

Type: Thailand. "Thailand, Khao Soidao, Iwatsuki et FukuokaT-7185" - KYO.

Plant very small, not more than $1 \mathrm{~cm}$ long. Rhizome long creeping, densely covered with brownish hairs. Stipe less than $3 \mathrm{~mm}$, slender than rhizome, with caducous hairs like those on rhizome, winged only at apex. Lamina simple, irregular in shape, bifid or trifid, elliptic, obcordate to oval, 0.5 $2.5 \mathrm{~cm}$ long, less than $0.5 \mathrm{~cm}$ wide; simple lamina or ultimate segments oblong-lanceolate, base cuneate round or cuneate, margin entire or way, apex rounded; with a simple distinct vein; many false veinlets, obliquely on the segment. Sori solitary, terminal on ultimate segments; involucres obconic or tubular, immersed, lips dilated, entire at margin. See Fig. 4 \& 9D.

Distribution in Pan-Himalaya: China (Xizang), Bhutan, E and C Nepal (Gandaki zone, Gorkha, Anbukhaireni, Deurali).

General distribution: China (Guangxi, Yunnan, SW Xizang), Myanmar (Moulmein, Merigu), Thailand. This species is critically endangered in Nepal by C. R. Fraser-Jenkins (2015).

Habitat: On moss-covered rocks in forests at lower mid altitudes.

Representative specimen examined: CHINA, XIZANG, South Xizang, Yarlung ZangboBrahmaputra area, Ganga Lake, CRFJ et A. B. FN 116 (PE).

4. Crepidomanes schmidtianum (Zenker ex Taschn.) K. Iwats., 1985, J. Fac. Sci. U. Tokyo III 13: 526; Iwatsuki, 1988, Bull. Univ. Mus. Univ. Tokyo 31: 251. ETrichomanes schmidtianum Zenker ex Taschn., 1843, Dissertation 34, pl. 1, f. 1, 3, 5; Copel., 1933, Phil. J, Sci. 51: 135, pl. 2, f. 1; Ghosh et al., 2004, Pterid. Fl. E. India 1: 244; Z. R. He, 2006, Fl. Yunnan. 20: 186. 三Vandenboschia schmidtiana (Zenker ex Taschner) Copel., 1938, Philipp. J. Sci. 67(1): 53; X. C. Zhang, 2008, Perspect. in Pteridophytes 33.

Type: India. "India, Nilgiris" - (K).
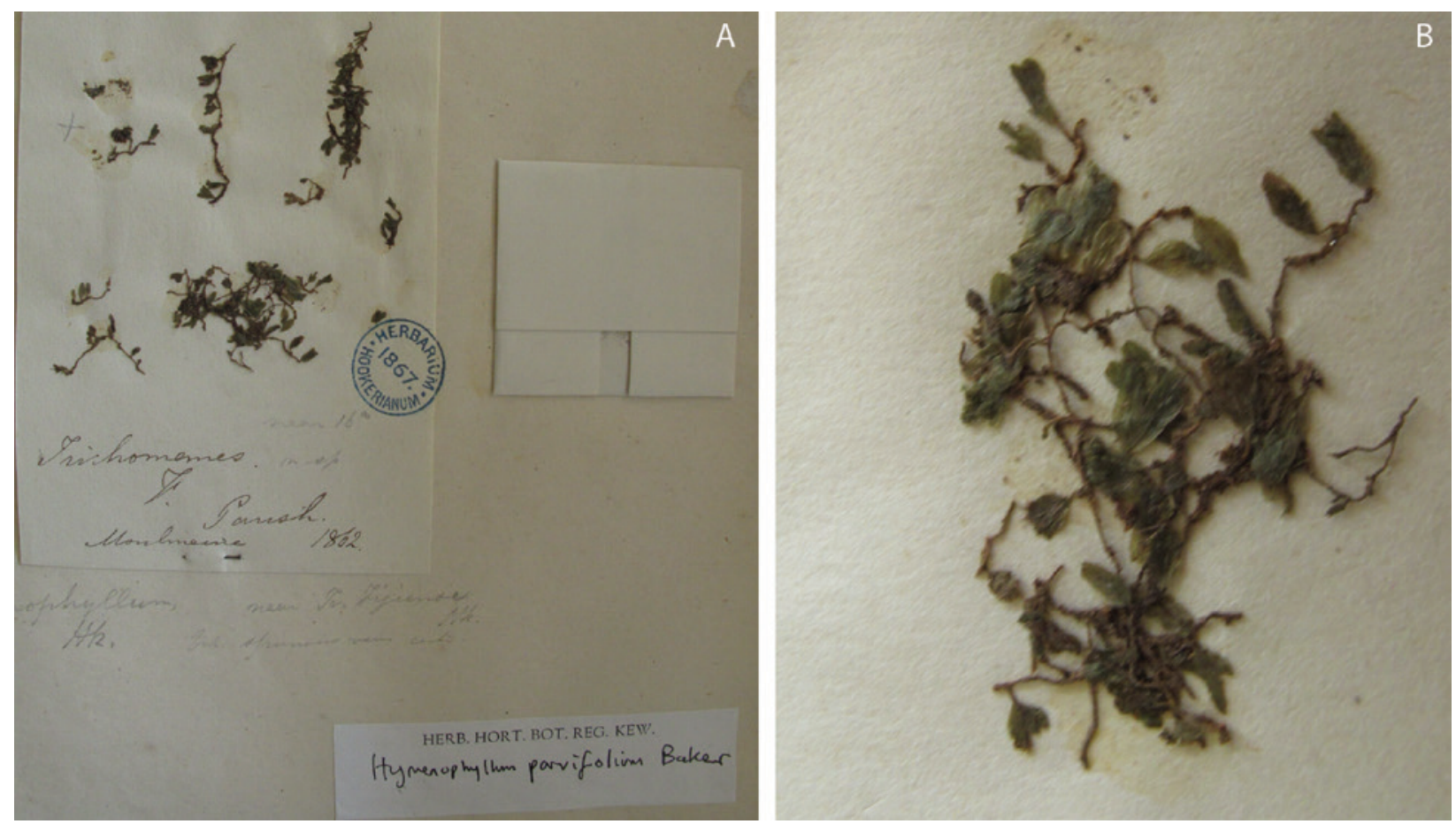

Fig. 4. Crepidomanes parvifolium (Baker) K. Iwats. A-B. Frond. A-B: Parish C. S. P. s. n. (K 001090172). 
= Vandenboschia titibuensis H. Itô, 1949, J. Jap. Bot. 24: 125, f. 1; K. Iwats., 1975, Fl. E. Himalaya 8: 172. $\equiv$ Lacosteopsis titubuensis (H. Itô) Nakaike, 1975, Enum. Pterid. Jap., Fil. 25. $\equiv$ Trichomanes titubuense (H. Itô) C.V. Morton, 1968, Contr. U.S. Natl. Herb. 38(5): 182. $\equiv$ Trichomanes titubuense (H. Itô) S. K. Wu, 1983, in C. Y. Wu, Fl. Xizangica 1: 52 , f. 13: 4, comb. superfl.

Type: Japan. "Iriyama in oppido Otakimura (Okutitiba), Prov. Musasi, Honsyu. H. Ito s.n., 20 VII 1949" - TI? (holotype).

= Trichomanes pyxidiferum L., 1753, Sp. Pl. 2: 1098 non, sens. Clarke, 1880, Trans. Linn. Soc. London, Bot. 1: 441; Bedd., 1883, Handb. Ferns Brit. India 42.

$=$ Trichomanes latifrons Bosch, 1863, Ned. Kruidk. Arch. 5(2): 209; Ghosh et al., 2004, Pterid. Fl. E. India 1: 244. $\equiv$ Crepidomanes schmidianum var. latifrons (Bosch) K. Iwats., 1985, J. Fac. Sci. Univ. Tokyo, Sect. 3, Bot. 13: 527. $\equiv$ Crepidomanes latifrons (Bosch) Ching, 1959, Fl. Reipubl. Popularis Sin. 2: 172. 1959. $\equiv$ Vandenboschia latifrons (Bosch) Copel., 1938, Philipp. J. Sci. 67(1): 104.

Type: India. "India orientalis, Khasiya, s.n. (Herb. Hook)" - K [K001090178] (holotype).

$=T$. cupressifolium Hayata, 1914, Icon. Pl. Formosan. 4: 136, f. 73.

Type: China. "Arisan, ad 8000-7000 ped. alt., B. Hayata et S. Sasaki, Jan., 1912” - TI?

Plants 3-6 cm tall. Rhizome ca. $0.5 \mathrm{~mm}$ in diam., densely covered with deep brown short hairs. Stipe remote, $0.8-2 \mathrm{~cm}$ apart, ca. $1 \mathrm{~cm} \times 03 \mathrm{~mm}$, wingless or narrowly winged throughout. Lamina bipinnatifid, dark green turn brown when dry, oblong-ovate, $2-4 \times$ 1-1.8 cm, membranous, glabrous, base cuneate, apex round; pinnae 4-10 pairs, closely spaced, imbricate, $1-1.4 \mathrm{~cm} \times 3-4 \mathrm{~mm}$, base oblique, apex obtuse or round; ultimate segments ca. $1.5 \mathrm{~mm}$ long, overlapped, oblong, acuminate at apex. Vein prominently raised on the surface, dark brown, free, alternate, hairy on lower surface, club-shaped, brown. Sori terminal on acroscopic segments; involucres tubular, narrowly winged, flaring at apex, lips usually dilated; receptacles long exserted. See Fig. $5 \& 10$ A.

Chromosome number: $2 n=72,108$ (Mehra and Singh, 1957; Mitui, 1968).

Distribution in Pan-Himalaya: China (Xizang), Bhutan, Nepal, N India (Subansiri).

General distribution: China (Guangxi, Taiwan, Xizang), Japan, Nepal, Bhutan, N India (Subansiri).

Habitat: On moist rocks or tree-trunks in shade area in forests at mid altitude $2000-3600 \mathrm{~m}$. Species delimitation is difficult to known throughout the Old world.

Representative specimen examined: CHINA, GUANGXI, Huangjiang, alt. 1500 m., Beijing Team 89-2831 (PE); XIZANG: Bomi, Yigong, alt. 2300 m., T. S. Ying et al. 497 (PE); YUNNAN: K. M. Feng 20748, 15.06.1936 (PE-00239529, C. latifrons), Chengkang, Snow Range, T. T. Yu 17008, 26.07.1938 (PE-00239530, C. latifrons).

2. Section Gonocormus (Bosch) K. Iwats.

Crepidomanes sect. Gonocormus (Bosch) K. Iwats., 1984, Acta Phytotax. Geobot. 35(4-6): 174. $\equiv$ Gonocormus Bosch, 1861, Verslagen Meded. Afd. Natuurk. Kon. Akad. Wetensch.: 321. $\equiv$ Trichomanes sect. Gonocormus (Bosch) Christ, 1897, Index Filic.: 27. $\equiv$ Trichomanes subg. Gonocormus (Bosch) C. Chr., 1906, Index Filic. XIV.

Lectotype: Gonocormus prolifer (Blume) Prantl (= Crepidomanes minutum (Blume) K. Iwats.).

Plant epiphytic or lithophytic. Rhizomes longcreeping, frequently branching, filiform, covered with dark brown hairs or glabrescent, roots absent, root-like shoots present. Lamina simple to quadripinnatifid, flabellate to narrowly ovate, stipe and rachis filamentous, much branched, dark, wiry, often proliferations often observed. Venation anadromous, false veinlets absent, internal cell walls thin and straight. Sori paratactic or pantotactic, campanulate, immersed in the apices of the segment, lips dilate, receptacles long-exserted.

One polymorphic species (Crepidomanes minutum (Blume) K. Iwats.) was found in PanHimalaya and throughout the Paleotropics.

Crepidomanes minutum (Blume) K. Iwats., 1985, J. Fac. Sci. Univ. Tokyo. Sect. 3, Bot. 13: 524; Iwatsuki, 1988, Bull. Univ. Mus. Univ. Tokyo 31: 251. E Trichomanes minutum Blume, 1828, Enum. Pl. Jav. 223; Copel., 1933, Phil. J. Sci. 51: 148; Holttum, 1954, Rev. Fl. Mal. 2: 96; Yoroi et K. Iwats., 1977, Acta Phytotax. Geobot. 28: 152. $\equiv$ Gonocormus minutus (Blume) Bosch, 1861, Hymen. Jav. 7. pl. 3; Copel., 1938, Phil. J. Sci. 67: 57; H. Ito, 1944, Fil. Jap. Ill. pl. 473; Ito, 1949, J. Jap. Bot. 24: 125; Ching, 1959, Fl. Reip, Pop. Sin. 2: 175, pl. 12, f. 1-6; Tagawa, 1959, Col. Ill. Jap. Pterid.44, 216. f. 68; Seriz., 1975, Sci. Rep. Takao Mus. N. H. 7: 17; Ghosh et al., 2004, Pterid. Fl. E. India 1: 241.

Type: Indonesia. "Java, Blume s.n.”- L. 
= Trichomanes proliferum Blume, 1828, Enum. Pl. Jav. 224; Hook., 1844, Sp. Fil. 1: 118. pl. 29B; Bedd, 1864, Ferns S. Ind. pl. 262; Copel., 1933, Phil. J. Sci. 51: 150; Tardieu et C. Chr., 1933, Fl. Gén. I.-C. 7(2): 66; Holttum, 1954, Rev. Fl. Mal. 2: 97. f. 33; Sledge, 1968, J. Linn. Soc. Bot. 60: 299. pl. 1. $\equiv$ Gonocormus prolifer (Blume) Prantl, 1875, Hymen. 51; Ching, 1959, Fl. Reip. Pop. Sin. 2: 178, pl. 12. f. 7.-

Type: Indonesia. "Java, Blume s.n." - L.

= Trichomanes diffusum Blume, 1828, Enum. P1. Jav. 225; Copel., 1933, Phil. J. Sci. 51: 149. $\equiv$ Gonocormus diffusus (Blume) Bosch, 1861, Hymen. Jav. 9, t. 4; Copel., 1938, Phil. J. Sci. 67: 57; K. Iwats., 1958, Acta Phytotax. Geobot. 17(6): 162.

Type: Indonesia. "Java, Blume s.n." - L.

$=$ Trichomanes saxifragoides C. Presl, 1843, Hymen. 39; Sledge, 1868, J. Linn. Soc. Bot. 60: 298. $\equiv$ Gonocormus saxifragoides (C. Presl) Bosch., 1861, Hymen. Jav. 9; H. S. Kung, 1988, Fl. Sichuan.
6: 139; Panigrahi et Sarn. Singh, 2005, Ferns FernAllies Arunachal Pradesh 1: 339.

Type: The Philippines. "Cuming plant. Exs. Philip. N. 256" - K [K000375720]; GH?, MICH?, L - [L0537118], [L0537119]; US - [US00134623]; $\mathrm{P}-$ [P00624459], [P00624458] (isotype).

= Trichomanes subpinnatifidum Bosch, 1861, Ned. Kruid. Arch. 5(2): 141; Bosch et Goddijn, 1913, Meded. Rijks-Herb. 17: 25. f. 14, 1913.

Type: Sri Lanka. "Ceylon, Gardner № 2" - L [L0052386] (holotype).

= Trichomanes teysmannii Bosch, 1861, Ned. Kruidk. Arch. 5(2): 142; Copel., 1933, Phil. J. Sci. 51: pl. 6. f. 1-3. $\equiv$ Gonocormus teysmanni (Bosch) Bosch, 1861, Hymen. Jav. 10; Tagawa et K. Iwats., 1967, Southeast As. St. 5: 40.

Type: Indonesia. "Sumatra, Teysmann"- L.

$=$ Trichomanes matthewii Christ, 1909, Not. Syst. 1: 56. $\equiv$ Gonocormus matthewii (Christ) Ching, 1959, Fl. Reip. Pop. Sin. 2: 177.
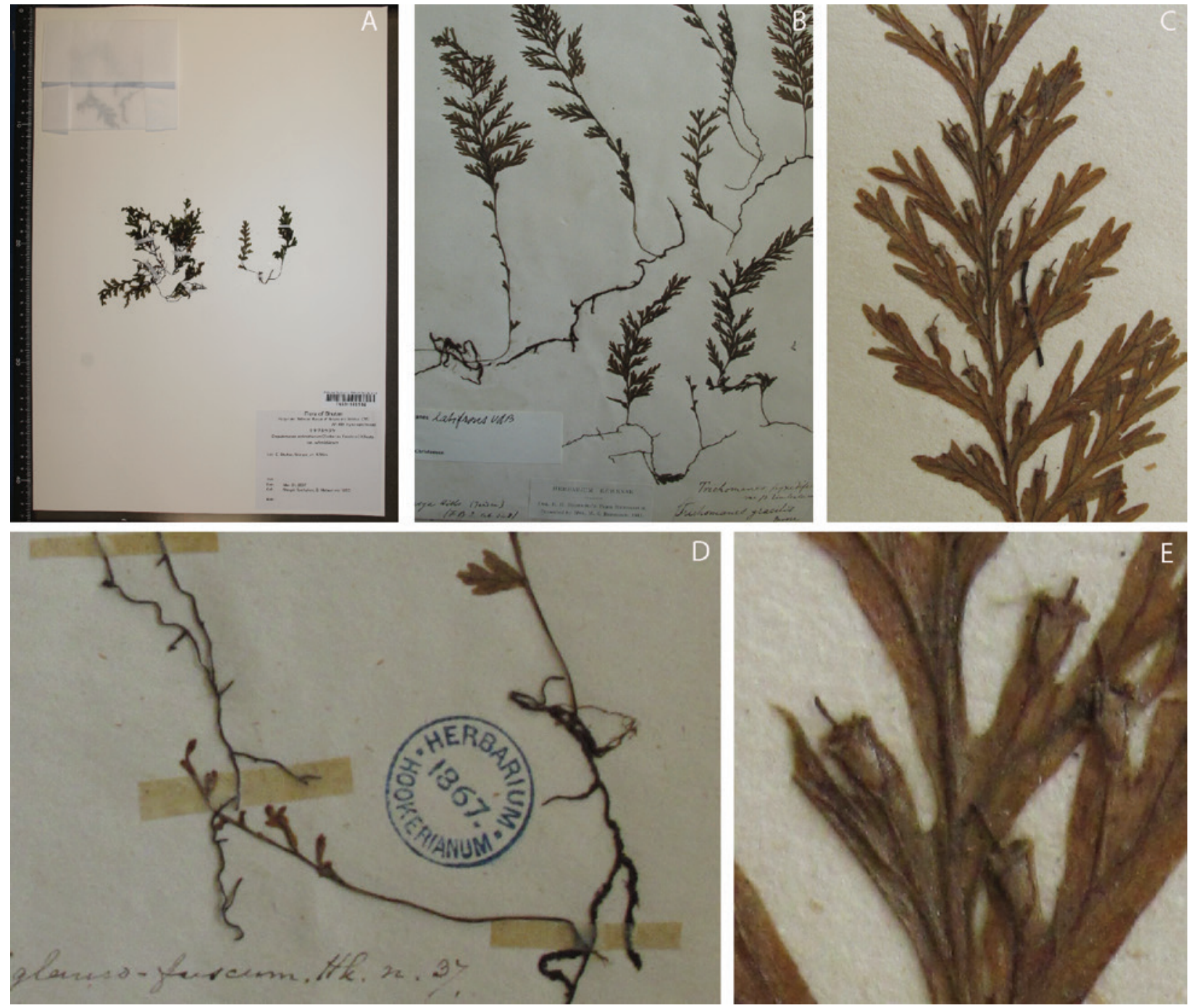

Fig. 5. Crepidomanes schmidtianum (Zenker ex Taschn.) K. Iwats. A-B. Frond, C. Part of frond, D. Rhizome, E. Sorus. A: W. Gyeltshen, S. Matsumoto 1226 (TNS 0118114); B-E: J. D. Hooker, T. Thomson sn. (K 001090178). 
Type: China. "China, Prov. Kwantung, North River Mong si Hap, 11.12.1907, Matthew C.G., s.n." - P [P00623412] (holotype), K- [K001090163; K001090162] (isotypes).

$=$ Trichomanes bonincola Nakai, 1926, Bot. Mag. Tokyo 40: 262. $\equiv$ Gonocormus bonincola (Nakai) Tagawa, 1951, J. Jap. Bot. 26: 186; Ohba, 1971, Sci. Rep. Tohoku Univ. 36: 83.

Lectotype: Japan. "Bonin, Chichijima, S. Nishimura 192" - TI, chosen by Iwatsuki 1985.

$=$ Trichomanes australe Ching, 1959, Acta Phytotax. Sin. 8: 137. 三 Gonocormus australe (Ching) Ching, 1959, Fl. Reip. Pop. Sin. 2: 176.

Type: China. "Hainan, Without exacte locality, E. Smith n. 1397" - PE [PE00042630!] (holotype); $\mathrm{BM}-[\mathrm{BM} 001044300]$ (isotype).

= Gonocormus siamensis Tagawa et K. Iwats, 1967, Acta Phytotax. Geobot. 22: 99. f. 3.

Type: Thailand. "Thailand, Khao Luang, Tagawa et al. T-4813" - KYO (holotype); K - [K001090207] (isotype).

- Trichomanes parvulum auct., non Poir.: Blume, 1828, Enum. Pl. Jav. 223; Bedd., 1866, Ferns Br. Ind. pl. 179; Makino, 1899, Phan. Pterid. Jap. Ill. 1: pl. 3; Ogata, 1931, Ic. Fil. Jap. 4: pl. 199; Copel., 1933, Phil. J. Sci. 51: 145. pl. 5; Tard. et C. Chr., 1939, Fl. Gén. I.-C. 7(2): 65.

Plants up to $5 \mathrm{~cm}$ long. Rhizomes long creeping, dark brown or blackish, filiform, much branched, $0.1-0.2 \mathrm{~mm}$ in diam., densely covered with dark brown hairs, root like shoot present. Stipe remote, filiform, $3-12 \times$ ca. $0.2 \mathrm{~mm}$, wingless or slightly narrowing wing only on the uppermost part, non auriculate, shortly unicellular ferruginous hairy throughout, sometimes occur proliferous on the stipe and rachis, proliferation repeated several times. Lamina simple, flabellate or ovate, $1-2 \times 1-1.5 \mathrm{~mm}$, base cordate or cuneate, palmate dissected into 3-5 shallowly or deeply lobed half way down or sometimes bipinnately divided; ultimate segments linear, $0.2-0.6 \mathrm{~mm}$ wide, apex obcordate. Lamina cell rectangular, oblong or sometimes irregular in shape; arranged in parallel row and cell wall thin and slightly warty; marginal cell elongate and irregular in shape, marginal cell walls slightly thick and straight. Vein palmate, free, forked, without false veinlet, the lower surface bearing minute, club-like hairs, reddish-brown, hairs more or less densely throughout. Sori terminal on the lobed, immersed, only few lobed fertile (2-4); involucres funnelform or campanulate, $1.5-2.5 \times 0.2-0.3 \mathrm{~mm}$, narrowing winged, lips flaring and dilated; receptacle long exseterd. See Fig. 6 \& 10B.
Chromosome number: $2 n=72,108,144$ (Bell, 1960; Braithwaite, 1975; Mitui, 1976; Yoroi et Iwatsuki, 1977).

Distribution in Pan-Himalaya: China (Sichuan: Emeishan, Nanchuan; Yunnan: Gongshan), Bhutan, $\mathrm{C}$ and E Nepal (rare).

General distribution: Africa, Madagascar, Russia (Far East), China (Anhui, Chongqing, Fujian, Guangxi, Guizhou, Hainan, Hunan, Jiangxi, Zhejiang, Taiwan, Sichuan, Yunnan), Japan, Korea, Bhutan, Nepal, India (Changlang, Assam, southern area), Sri Lanka, Thailand, Vietnam, Cambodia, The Philippines, Malaysia, Indonesia (Java), Johanna Islands and the Moluccas, Australia (Queensland, N. S. Wales), Pacific Islands (Melanesia, Micronesia, Polynesia).

Habitat: Tree trunks or on rocks in forests and valley or wet rocks near streams, on moos-covered at low mid altitude.

Note: This species is one of the complex species of the Hymenophyllaceae, so many authors gave name based on the phonetic features.

Representative specimen examined: CHINA, SICHUAN, Emeishan: 600 m, K. H. Shing et K. Y. Lang 1897 (PE, Crepidomanes minutus). $700 \mathrm{~m}, \mathrm{~K} . \mathrm{H}$. Shing et K. Y. Lang 18060 (PE, C. minutus). 800 $\mathrm{m}, \mathrm{X}$. J. Zheng 10085 (PE). Nanchuan: $650 \mathrm{~m}, \mathrm{Z}$. Y. Liu 18060 (Paratype PE, C. minutus). 900 m, Z. Y. Liu 3836 (PE, C. minutus). YUNNAN, Gongshan: Tibetan Exp. Team 9783 (PE, C. australe).

\section{VANDENBOSCHIA}

Vandenboschia Copel. 1938, Philipp. J. Sci. 67(1): 51. $\equiv$ Trichomanes subg. Vandenboschia (Copel.) Allan, 1961, Fl. New Zealand: 34.

Type: Vandenboschia radicans (Sw.) Copel. (= Trichomanes radicans $\mathrm{Sw}$.

Key to the subgenus

1. Lamina bipinnate to 5-pinnatifid, ovate to linear-ovate 1. Vandenboschia

+ Lamina pinnate with symmetric pinnae, elliptic 2. Lacosteopsis

\section{1. Subgenus Vandenboschia}

Plants epiphytic or epilithic. Rhizomes shortor long-creeping, irregularly branching, rather thick, densely covered with brown to bright brown multicellular hairs, roots numerous and robust. Lamina bipinnate to 5-pinnatifid, ovate to linearovate, venation anadromous, false veinlets absent, laminae often reduced, regular arrangement of elongate cells observed in some species, internal cell walls various (thin to thick, straight to coarsely 
pitted). Sori paratactic, tubular to campanulate, lips sometimes dilate, receptacles long-exserted.

One species was found in Pan-Himalaya and more than 15 species throughout the tropics, extending to northern temperate regions.

1. Vandenboschia striata (D. Don) Ebihara, 2013, Fl. China, Vol. 2-3: 109. 三 Trichomanes striatum D. Don, 1825, Prodr. Fl. Nepal. 11; H. S. Kung, 1988, Fl. Sichuan. 6: 158; Ghosh et al., 2004, Pterid. Fl. E. India 1: 246; Z. R. He, 2006, Fl. Yunnan. 20: 185.

Type: Nepal. "Nepalia, Hamilton s.n." - BM [BM001044294] (holotype).

= Trichomanes naseanum Christ, 1905, Bull. Soc. Bot. Fr. Mém. 1: 11; Ogata, 1928, Ic. Fil. Jap. 1: pl. 46; Tardieu et C. Chr., 1939, Fl. Gén. I.-C. 7(2): 71. $\equiv$ Trichomanes radicans var. naseanum (Christ)
Lelling., 1968, Amer. Fern J. 58(4): 157; K. Iwats., 1985, J. Fac. Sci. Univ. Tokyo, Sect. 3, 13: 528. $\equiv$ Vandenboschia naseana (Christ) Ching, 1939, Acta Phytotax. Sin. Gén. I.-C. 7(2): 71; Ching, 1959, Fl. Reip. Pop. Sin. 2: 186; Serizawa, 1975, Sci. Rep. Takao Mus. N. H. 7: 23. $\equiv$ Vandenboschia radicans var. naseanum (Christ) H. Itô, 1949, J. Jap. Bot. 24: 124; Tagawa, 1959, Col. Ill. Jap. Pterid. 45, 260, f. 69b; Iwatsuki, 1988, Bull. Univ. Mus. Univ. Tokyo 31: 251. $\equiv$ Crepidomanes radicans var. naseanum (Christ) K. Iwats., 1985, J. Fac. Sci. Univ. Tokyo, Bot. 13(5): 530. $\equiv$ Lacosteopsis orientalis var. naseana (Christ) Nakaike, 1975, Enum. Pterid. Jap. 24.

Type: China. "Su-tchuen [Sze-ch'uan], Mount Omei, 3000 m alt., Faber 102" - P [P00623993; P00623994] (syntypes); "Ryukyu, Oshima, Coll. Unknown Christ det. 1" - TI (lectotype).
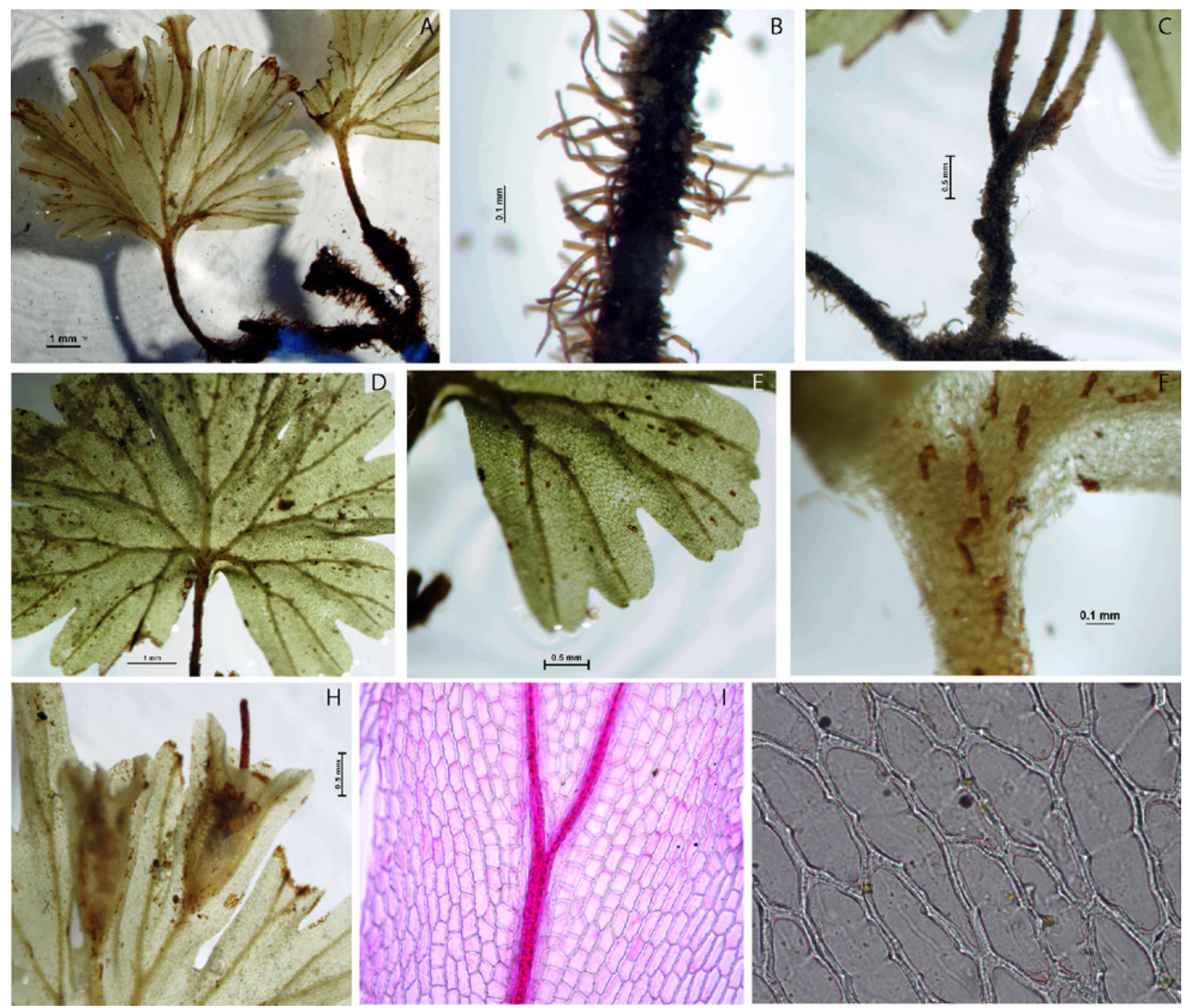

Fig. 6. Crepidomanes minutum (Blume) K. Iwats. A. Frond, B. Rhizome with hairs, C. Stipe with proliferation, D. Lamina E. Ultimate segment F. Rachis with club-shaped brown hairs, H. Sorus, I. Lamina cell in outline, J. Lamina cell. A - J: Tibetan Exp. Team 9783. (PE 00239562). 
= Crepidomanes birmanicum (Bedd.) K. Iwats., 1985, J. Fac. Sci. Univ. Tokyo, Sect. 3, Bot, 13: 530. $\equiv$ Trichomanes birmanicum Bedd., 1876, Ferns Br. Ind. Suppl. 3. pl. 349; Tardieu et C. Chr., 1939, Fl. Gén. I.-C. 7(2): 71; Tagawa et K. Iwats., 1979, Fl. Thail. 3: 84. f. 5-7; Z. R. He, 2006, Fl. Yunnan. 20: 186 . $\equiv$ T. radicans var. birmanicum (Bedd.) C. Chr., 1906, Ind. Fil. 636. $\equiv$ Vandenboschia birmanica (Bedd.) Ching, 1959, Acta Phytotax. Sin. 8: 135; Ching, 1959, Fl. Reip. Pop. Sin. 2: 185.

Type: Myanmar. "Maulmein, Parish 181" - K [K001090197, K001090198] (syntypes).

= Trichomanes somae Nakai, 1926, Bot. Mag. (Tokyo) 40. 267.

Type: China. "Formosa: Rimogan, T. Soma s.n." - TI (?).

Plants $10-60 \mathrm{~cm}$ tall. Rhizome dark brown nearly blackish, $1-1.2 \mathrm{~mm}$ in diam., densely covered with dark brown multicellular hairs, rootlets with sparse hairy. Stipes terete, remote, $1-3 \mathrm{~cm}$ apart, light brown, 5-8 $\mathrm{cm} \times 0.8-1 \mathrm{~mm}$, glabrous, broadly winged nearly to base, wing more than $0.5 \mathrm{~mm}$ broad on both side, entire and flat. Rachis and costae stramineous, two types of hairs present, sparsely with club-shaped brownish hairs throughout and base of the costae with long multicellular hairs, broadly winged throughout, entire and flat. Lamina tripinnatifid, oblong-lanceolate, 10-45 × 3-6 cm, membranous, glabrous, apex acute, base round to cordate; pinnae 9-14 pairs, alternate, subsessile or stalk shortly winged, subdeltoidto oblique ovate, $1-2.8 \times 0.5-2 \mathrm{~cm}$, base unequally cuneate, apex moderately acute; ultimate segments simple or forked, narrowly linear, $1.5-3 \times 0.4-0.7 \mathrm{~mm}$, with 1 (or 2) veinlets, entire, apex rounded, slightly lobed. Lamina cell polygonal, irregular in shaped; cell wall thin and straight; marginal cell walls irregular in shaped, thin and straight. Veins dichotomous, dark green-brown, obviously raised on each surface, sparsely with club-shaped brown hairs. Sori on upper parts of fronds, apical on short acroscopic segments; involucres tubular, $1.7-2 \times 1-1.3 \mathrm{~mm}$, lips truncate and entire or slightly dilated at mouth, narrowly winged, lobes slightly constricted below base of involucre; receptacles long exserted, brown, filiform. See Fig. 7 \& 10C.

Chromosome number: $2 n=72$ (Mitui, 1975).

Distribution in Pan-Himalaya: China (Yunnan: Gongshan; Xizang: Medog, Nyinchi), India (Sikkim, Darjeeling, Kameng, Subansiri, Debang Valley, Changlang, Tirap, Pithoragarh), W, C and E Nepal, Bhutan.
General distribution: China (Guangdong, Guangxi, Guizhou, Hainan, Taiwan, Sichuan, Yunnan), Japan (Ryukyu), Bhutan, W, C and E Nepal, India (Changlang, Darjeeling, Kameng, Subansiri, Debang Valley, Tirap, Pithoragarh, Meghalaya, Sikkim), Myanmar, Laos, Thailand, Vietnam.

Habitat: On wet and muddy rocks usually along stream or on moist surfaces of cliffs in deep shade in forest $700-2000 \mathrm{~m}$.

Representative specimen examined: CHIAN, YUNNAN, Gongshan: From Ba-po to Kongdang: $1450 \mathrm{~m}, \mathrm{X}$. H. Jin et al. DLJ-ET 0665 (PE, Vandenboschia naseanum). 1400 m, Tibetan Exped. Team 9335 (PE, Trichomanes stratia). XIZANG, Medog: Qinghai-Tibet Team (Vegetation Group) 3168 (PE, T. stratia); Beibeng: $900 \mathrm{~m}$, South Tiebt Exped. Team (STET) STET2256 (PE, T. stratia). Nyinchi: 2400 m, B. S. Li et S. Z. Cheng 1896 (PE, T. stratia). $1400 \mathrm{~m}, \mathrm{~B}$. S. Li et S. Z. Cheng 1499 (PE, T. stratia).

2. Subgenus Lacosteopsis (Prantl) Ebihara et K. Iwats., 2006, Blumea, 51(2): 242. 三 Trichomanes sect. Lacosteopsis Prantl, 1875, Unters. Morph. Gefasskrypt.: 53. $\equiv$ Lacosteopsis (Prantl) Nakaike, 1975, Enum. Pterid. Jap., Fil. 21.

Lectotype: Trichomanes luschnatianum C. Presl (= Vandenboschia rupestris Ebihara et K. Iwats., 2006, Blumea, 51(2): 242. [= Hymenophyllum rupestre Raddi]), chosen by Christensen (1906).

Rhizomes long-creeping, frequently branching, sparsely to densely covered with brown hairs, mostly climbing with rootless. Stipes clustered or irregularly distanced. Blades usually oncepinnate with symmetric pinnae, elliptic, venation anadromous, false veinlets absent, internal cell walls slightly thick and wavy. Sori paratactic, tubular, lips truncate or sometimes dilate, receptacles longexserted.

Vandenboschia auriculata (Blume) Copel., 1938, Phil. J. Sci. 67: 55; H. Ito, 1944, Fil. Jap. Ill. pl. 471; Ching, 1959, Fl. Reip. Pop. Sin 2: 181. pl. 13. f. 1-2; Tagawa, 1959, Col. Ill. Jap. Pterid. 46, 260, f. 73; De Vol, 1975, Fl. Taiwan 1: 114; K. Iwats., 1975, Fl. E. Himalaya 8: 172; Seriz., 1975, Sci. Rep. Takao Mus. N. H. 7: 22. $\equiv$ Trichomanes auriculatum Blume, 1828, Enum. Pl. Jav. 225; Bosch, 1861, Hymen. Jav., t. 25; Bedd., 1883, Ferns Br. Ind. pl. 182; Makino, 1899, Phan. Pterid. Jap. Ill. 1: pl. 22; Ogata, 1931, Ic. Fil. Jap. 4: pl. 198; Copel., 1933, Phil. J. Sci. 51: 223; Tardieu et C. Chr., 1939, 
Fl. Gén I.-C. 7(2): 69; Holttum, 1954, Rev. Fl. Mal. Iwatsuki, 1988, Bull. Univ. Mus. Univ. Tokyo 31: 2: 105, f. 41; Tagawa et K. Iwats., 1979, Fl. Thail. 251. $\equiv$ Lacostea auriculata (Blume) Prantl, 1875, 3: 83; Ghosh et al., 2004, Pterid. Fl. E. India 1: 245; Hymen. 50. $\equiv$ Lacosteopsis auriculata (Blume) Z.R. He, 2006, Fl. Yunnan. 20: 183. $\equiv$ Cephalomanes Nakaike, 1975, Enum. Pterid. Jap. 21; Panigrahi et auriculatum (Blume) Bosch, 1859, Ned. Kruid. Sarn. Singh, 2005, Ferns Fern-Allies Arunachal PraArch. 4: 352; Bosch, 1861, Hymen. Jav. 34, t. 25; desh 1:344. $\equiv$ Crepidomanes auriculatum (Blume)
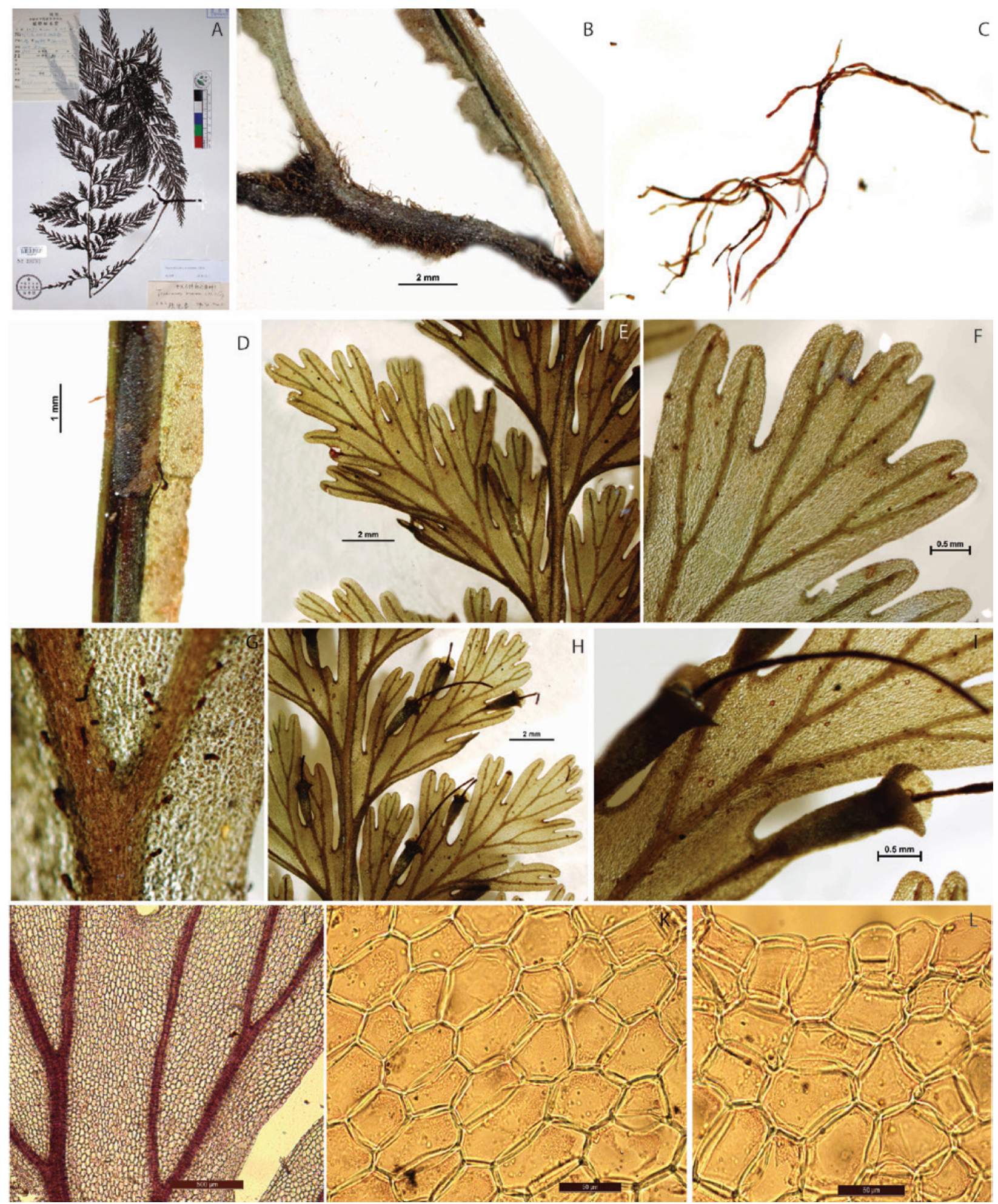

Fig. 7. Vandenboschia striata Baker A. Frond, B. Rhizome and stipe, C. Rhizome hair, D. Stipe winged, E. Part of frond, F. Ultimate segments, G. Vein with club-shaped hairs, H. Fertile frond, I. Sorus, J. Pinna cell in outline, K. Lamina cells, L. Marginal cells. A-L: K. H. Shing, Y. X. Lin et X. C. Zhang s. n. (PE 01347071). 


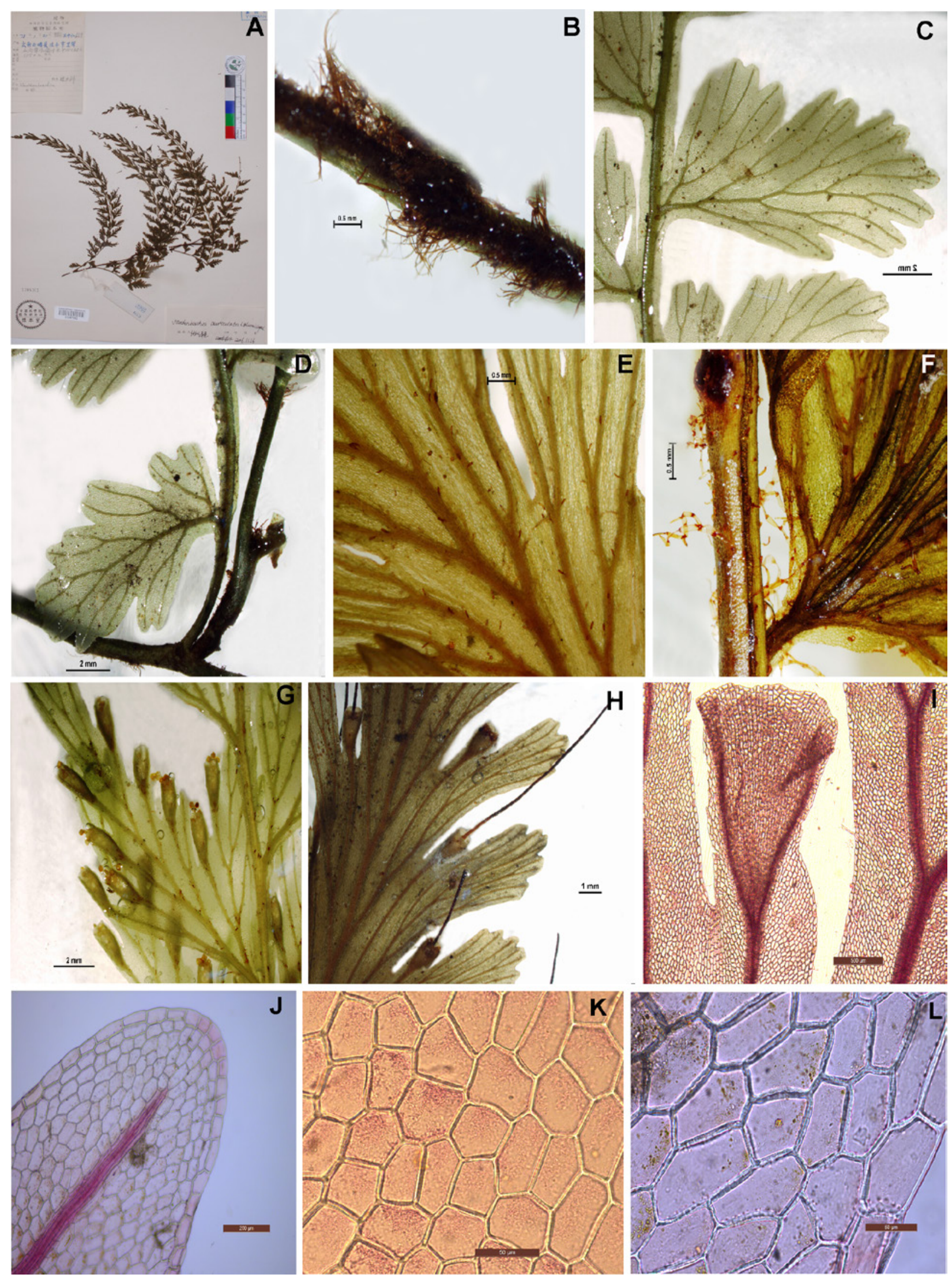

Fig. 8. Vandenboschia auriculata (Blume) Copel. A. Frond, B. Rhizome, C. Rachis and pinna, D. Part of frond, E. Vein with hairy, F. Rachis and pinna base, G. Fertile frond, H. Sorus and receptacle, I. Sorus and pinna cell in outline, J. Tips of pinna, K. Lamina cells, L. Marginal cells. A-L: Z. R. Wang 669 (PE 01267893). 
K. Iwats., 1985, J. Fac. Sci. Univ. Tokyo, Sect. 3, 13: 528; H.S. Kung, 1988, Fl. Sichuan. 6: 157.

Type: Indonesia. "Java, Blume s. n." - L.

= Trichomanes dissectum J. Sm., 1841, Hook. J. Bot. 3: 417; Hook., 1846, Sp. Fil. 1: 140, n.n.; Bedd., 1866, Ferns Br. Ind. pl. 182.

Type: The Philippines. "Luzon, Cuming 129" - [GOET009201; GH00022223; K000375471; K000375472; L0544637; K000375538; L0544638; LE00007930; LE00007929; P00624497; P00624498; P00624499;] (isotypes).

= Trichomanes miyakei Yabe, 1905, Bot. Mag. Tokyo 19: 34; Copel., 1933, Phil. J. Sci. 51: 222.

Type: China. "Taiwan, Rahao, K. Miyake s. n." - TI (holotype).

$=$ Vandenboschia hainanensis Ching et P. S. Chiu, 1959, Acta Phytotax. Sin. 8: 135. pl. 18. f. 12; Ching, 1959, Fl. Reip. Pop. Sin. 2: 180.

Type: China. "Hainan, Chim Fung Ling, S. K. Lau 3813" - PE [PE00042653!] (holotype).

Plants $12-30 \mathrm{~cm}$ tall. Rhizome long creeping, climbing to several meters on tree trunk, stramineous to dark brown, $1-1.8 \mathrm{~mm}$ in diam., compressed, stiff, densely covered with hairs, dark brown, multicellular, twisted, caducous. Stipes terete, remote, 3-5 $\mathrm{cm}$ apart, terete, stramineous or light to deep brown, $0.4-1.3 \mathrm{~cm} \times 0.5-1 \mathrm{~mm}$, multicellular hairs throughout, wingless or narrowly winged on upper part. Rachis stramineous, very narrowly winged or nearly wingless throughout, with multicellular hairs. Lamina pinnate, linearlanceolate, $15-35 \times 3-4 \mathrm{~cm}$, base cuneate, apex acuminate, densely with hairy at base, narrowing towards base; pinnae 15-28 pairs, closely spaced, alternate, sessile, glabrous, rhomboid or obovate to oblong, $1.2-1.8 \times 0.7-1.5 \mathrm{~cm}$, apex rounded to obtuse, margin slightly or deeply lobed, lobes round or slightly acute with 1 or 2 veilets, base unequal, base truncate acroscopically and cuneate basiscopically. Lamina cell small, polyhedral, irregular in shaped; cell walls thick and straight; marginal cell elongate, slightly thick and straight. Veins dichomotous branching, free, forked, green or stramineous to light reddish-brown, sparsely hairy throughout, hairs club-shaped, brown to reddishbrown. Sori apical on the segments, $10-14$ per pinna; involucres narrowly tubular, $2.1-2.3 \times 0.6-0.9 \mathrm{~mm}$, lips truncate, dialate, narrowly winged; receptacles long exserted, slender, curved, sometimes more than $4 \mathrm{~mm}$ long. See Fig. 8 \& 10D.

Chromosome number: $2 n=72$ (Mitui, 1966, 1975).
Distribution in Pan-Himalaya: China (Yunnan: Gongshan; Xizang: Medog), C and E Nepal, Bhutan, India (Sikkim, Darjeeling, Kameng, Subansiri, Siang, Tirap, Changlang).

General distribution: China (Guangdong, Guangxi, Guizhou, Hainan, Jiangxi, Taiwan, Zhejiang, Sichuan, Xizang, Yunnan), Japan, Bhutan, $\mathrm{C}$ and $\mathrm{E}$ Nepal, Myanmar, NE India (Himalayas, Sikkim, Darjeeling, Kameng, Subansiri, Siang, Tirap, Changlang), Laos, Thailand, Cambodia, Malaysia, The Philippines, Indonesia, Pacific Islands (Micronesia).

Habitat: Creeping on tree trunks or rarely on rock surface in dense forests usually in lowlands at 500-2700 m.

Representative specimen examined: CHINA, YUNNAN, Gongshan: $2000 \mathrm{~m}$, Tibetan Exped. Team 8089 (PE, Vandenboschia auriculata). 1900 m, Tibetan Exped. Team 8983 (PE, V. auriculata). 1700 m, Tibetan Exped. Team 9132 (PE, V. auriculata). 1600 m, Tibetan Exped. Team 9132 (PE, V. auriculata). $2000 \mathrm{~m}, \mathrm{C}$. W. Wang 67248 (PE, T. auriculatum). XIZANG, Medog: $1500 \mathrm{~m}$, B. S. Li et S. Z. Cheng 2903 (PE, T. auriculatum). $1700 \mathrm{~m}, \mathrm{~B} . \mathrm{S}$. Li et S. Z. Cheng 2703 (PE, T. auriculatum). $1750 \mathrm{~m}$, Tibetan Exped. Team 74-4414 (PE, T. auriculatum). 1800 m, B. S. Li et S. Z. Cheng 4311 (PE, T. auriculatum). $1500 \mathrm{~m}$, B. S. Li et S. Z. Cheng 4495 (PE, T. auriculatum). $950 \mathrm{~m}$, Ecological Team Plateau Group 11048 (PE, T. auriculatum). $1800 \mathrm{~m}$, Ecological Team Plateau Group 11447 (PE, T. auriculatum). 1800 m, Qinghai-Tibet Team (Vegetation Group) 2933 (PE, V. auriculata); Beibeng hills, $1900 \mathrm{~m}$, South Tibet Exped. Team (STET) STET2270 (PE, V. auriculata). BHUTAN, s. loc., 2020 m, B. Bartholomew 3769 (PE, Trichomanes auriculatum).

\section{Acknowledgements}

We wish to express sincere thanks to the curators and supporting staffs of PE, K, E and BM herbaria for their kind permission to study the specimen and use facilities. We would like to express the gratitude to Mr. Christopher Fraser-Jenkins and Nawal Shrestha for providing relevant literature and information and also extend to Dr. Ebihara for providing pictures of specimens and valuable advices. Special thanks to Professor Zenith Oo and Mr. Christopher Fraser-Jenkins for their valuable suggestions. The work was supported by the CASTWAS postdoctoral Fellowship program, 2012 (FR number: 3240267233). 

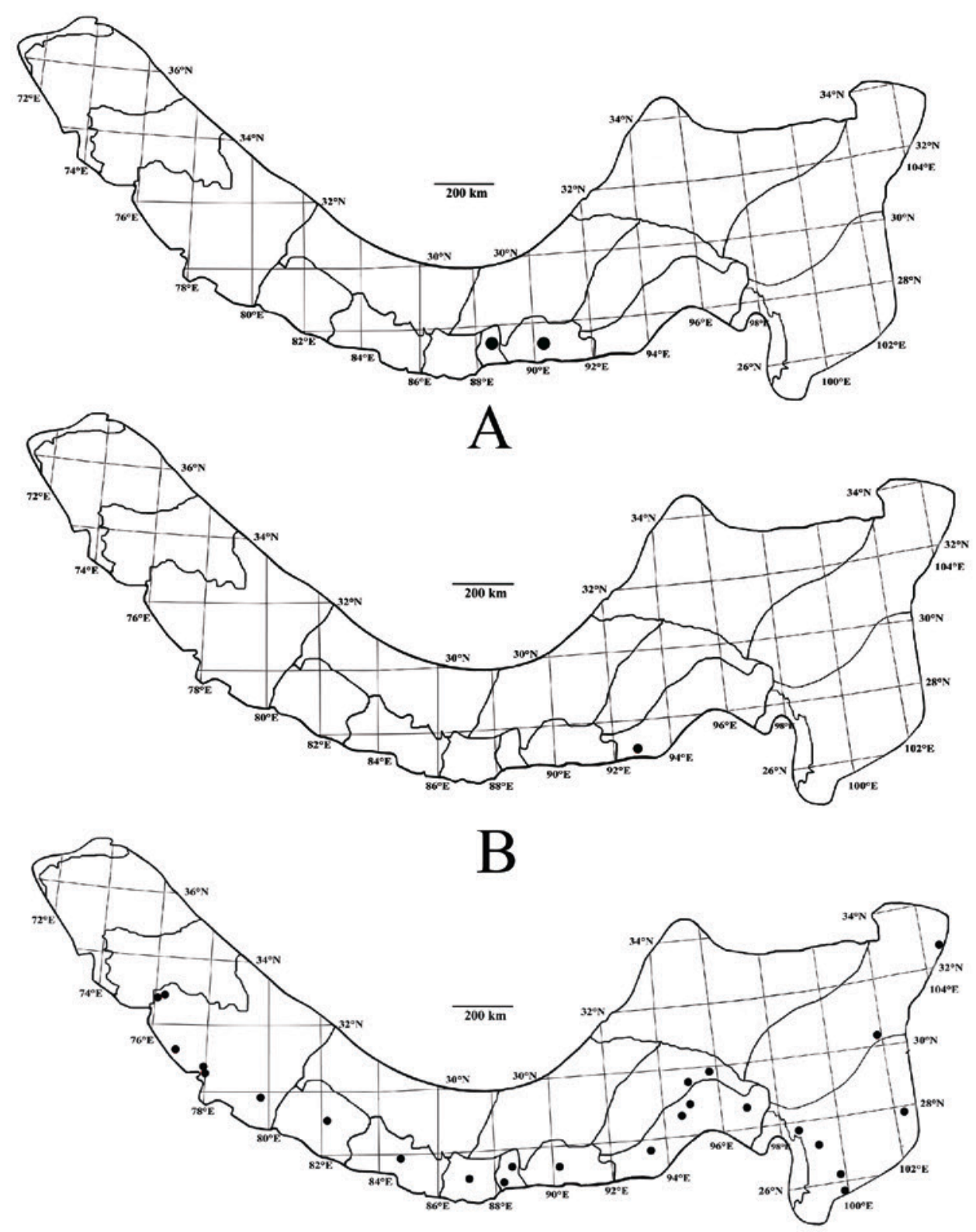

$\mathrm{C}$

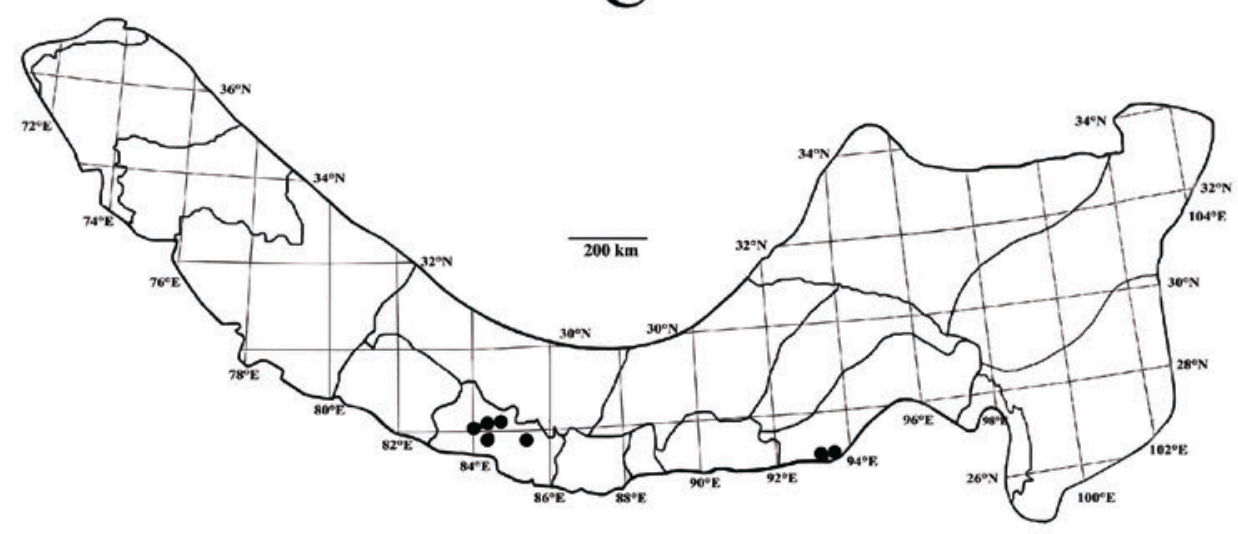

$\mathrm{D}$

Fig. 9. Distribution of the Didymoglossum and Crepidomanes in Pan Himalaya regions: a - Didymoglossum sublimbatum (Müll. Berol.) Ebihara et K. Iwats.; b - Crepidomanes bipunctatum (Poir.) Copel.; c - Crepidomanes latealatum (Bosch) Copel.; d - Crepidomanes parvifolium (Baker) K. Iwats. 

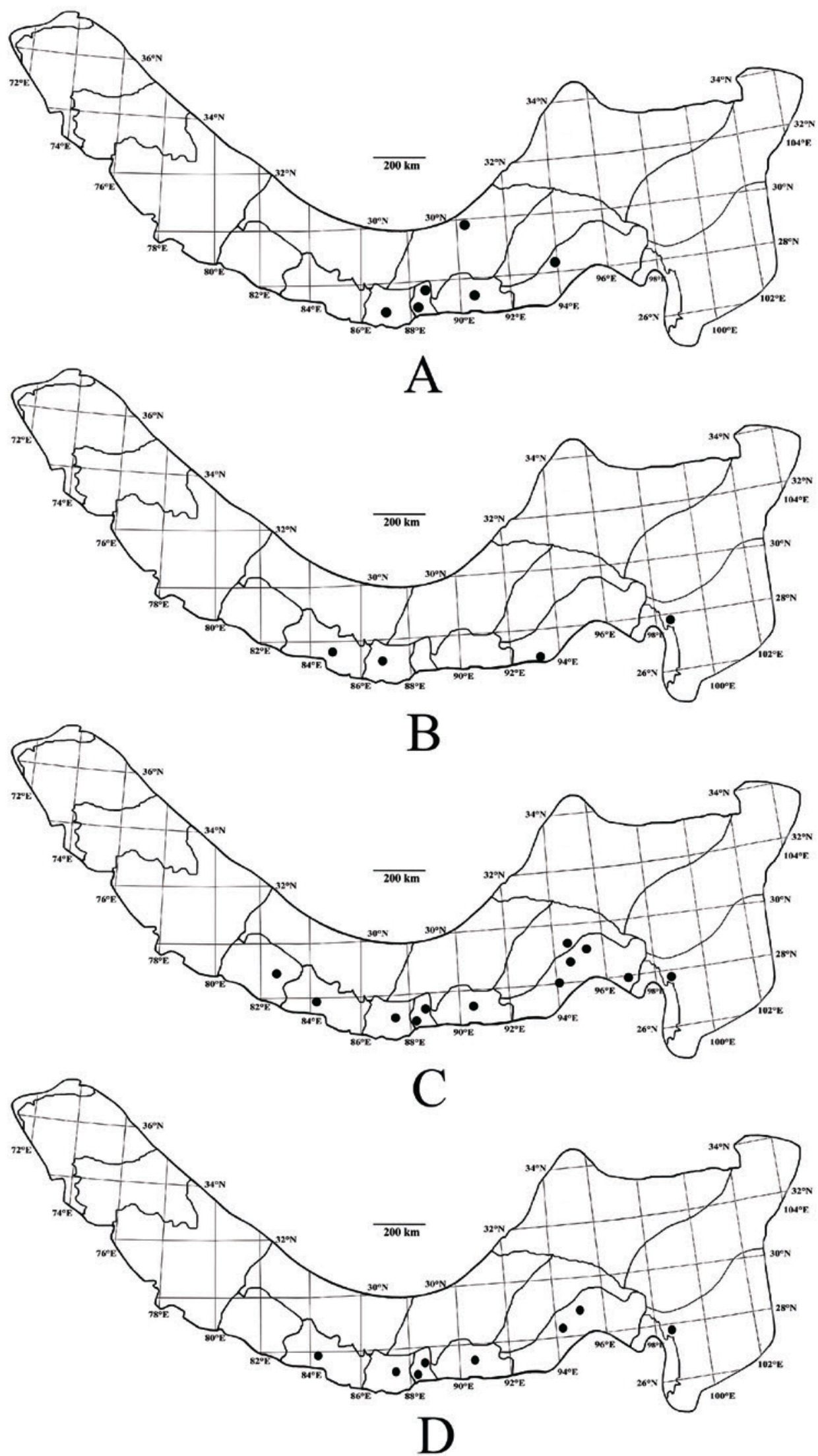

Fig. 10. Distribution of the Crepidomanes and Vandenboschia in Pan Himalaya regions: a - Crepidomanes schmidtianum (Zenker ex Taschn.) K. Iwats.; b - Crepidomanes minutum (Blume) K. Iwats.; c - Vandenboschia striata (D. Don) Ebihara; d - Vandenboschia auriculata (Blume) Copel. 


\section{REFERENCES / ЛИTEPATУРA}

Bell P. R. 1960. The morphology and cytology of sporogenesis of Trichomanes proliferum Bl. New Phytologist 59: 53-59.

Bosch R. B. v. d. 1859. Synopsis Hymenophyllacearum: monographiae hujus ordinis prodromus. Ned. Kruidk. Arch. 4: 341-419.

Bosch R. B. v. d. 1861. Hymenophyllaceae Javanicae. Akad. van Wetenschappen, Amsterdam, 64 pp.

Braithwaite A. F. 1975. Cytotaxonomic observations on some Hymenophyllaceae from the New Hebrides, Fiji and New Caledonia. Botanical Journal of the Linnean Society 71: 167-189.

Ching R. C. 1959. Hymenophyllaceae. In: Flora Reipublicae Popularis Sinicae. Ed. R. C. Ching. Science Press, Beijing, 2: 136-96.

Copeland E. B. 1933. Trichomanes. Philipp. J. Sci. 51: 119-280.

Copeland E. B. 1938. Genera Hymenophyllacearum. Philipp. J. Sci. 67: 1-110.

Dixit R. D. 1984. A census of the Indian Pteridophytes. In: Flora of India. Series 4. Botanical survey of India, Howrah, $177 \mathrm{pp}$.

Ebihara A., Dubussion J-Y., Iwatsuki K., Hennequin S., Ito M. 2006. A taxonomic revision of Hymenophyllaceae. Blumea 51: 221-280. DOI: 10.3767/000651906X622210

Fraser-Jenkins C. R. 2012. Rare and Threatened Pteridophytes of Asia 2. Endangered Species of India - the Higher IUCN Categories. Bull. Natl. Mus. Nat. Sci., Ser. B 38(4): 153-181.

Fraser-Jenkins C. R., Kandel D. R., Pariyar S. 2015. Ferns and fern-allies of Nepal. Vol. 1. National Herbarium and Plant Laboratories, Department of Plant Resources, Ministry of Forests and Soil Conservation, Kathmandu, Nepal, 508 pp.

Ghosh S. R., Ghosh B., Biswas A., Ghosh R. K. 2004. The Pteridophytic Flora of Eastern India 1. Flora of India. Series 4. Botanical survey of India, Kolkata, $591 \mathrm{pp}$.

He Z. R. 2006. Hymenophyllaceae. In: Flora Yunnanica. Vol. 20 (Pteridophyta). Ed. Z. Wu. Science Press, Beijing, $785 \mathrm{pp}$.

Holttum R. E. 1954. A revised Flora of Malaya Vol. 2. Ferns of Malaya. Government Printing Office, Singapore, $653 \mathrm{pp}$.

Ito H. 1949. Hymenophyllaceae of Japan. Journal of Japanese Botany 24: 124-127.

Iwatsuki K. 1958a. Taxonomic Studies of Pteridophyta I. Acta Phytotax. Geobot. 17(4): 65-72.

Iwatsuki K. 1958b. Taxonomic Studies of Pteridophyta II. Acta Phytotax. Geobot. 17(6): 161-166.

Iwatsuki K. 1975. Pteridophyta. In: Flora of Eastern Himalaya. Ed. H. Ohashi. The University Museum, the University of Tokyo, Tokyo, 8: 166-205.

Iwatsuki K. 1985. The Hymenophyllaceae of Asia, excluding Malesia. J. Fac. Sci. Univ. Tokyo, Sect. 3, Bot. 13: 501-551.

Iwatsuki K. 1988. An enumeration of the pteridophytes of Nepal. In: The Himalayan Plants. Eds. H. Ohba, S. B. Malla. Bull. Univ. Mus. Univ. Tokyo 31: 231-339. http://dx.doi.org/10.3767/000651906X622210

Iwatsuki K. 1990. Hymenophyllaceae. In: The families and genera of vascular plants. The families and genera of vascular plants. Pteridophytes and Gymnosperms. Ed. K. Kubitzki. Springer Verlag, Berlin, 1: 157-163 pp.

Kung H. S. 1988. Flora Sichuanica. T. 6. Pteridophyta. Chengdu, 396 pp.

Liu J. X., Zhang Q. Y., Ebihara A., Iwatsuki K. 2013. Hymenophyllacae Mart. In: Flora of China. Vol. 2-3. (Pteridophytes). Eds. Z. Y. Wu, P. H. Raven, D. Y. Hong. Science Press, Beijing et Missouri Botanical Garden Press, St. Louis, (2-3): 93-109.

Makino T. 1899. Plantae Japonenses novae vel minus cognitae. Bot. Mag. (Tokyo) 13: 44-48.

Mehra P. N., Singh G. 1957. Cytology of Hymenophyllaceae. Journal of Genetics 55: 379-393.

Mitui K. 1966. Chromosome studies on Japanese ferns (2). Journal of Japanese Botany 41: 60-64.

Mitui K. 1975. Chromosome numbers of Japanese pteridophytes. Bull. Nippon Dental College, General Educ. 4: 221-271.

Morton C. $\boldsymbol{V}$. 1968. The genera, subgenera and sections of Hymenophyllaceae. Contr. U Nat. Herb. 38: $153-201$.

Nakaike T. 1975. Enumeratio Pteridophytarum Japonicarum - Filicales. University of Tokyo Press, Tokyo, 375 pp. Nwe T. Y., Zhang X.-C. 2017. Taxonomic studies of lycophytes and ferns from the Pan-Himalaya (I): Hymenophyllum (Hymenophyllaceae). Turczaninowia 20, 2: 75-96. DOI: 10.14258/turczaninowia.20.2.7

PPG I. 2016. A community derived classification for extant lycophytes and ferns. J. Syst. Evol. 54(6): 563-603.

DOI: $10.1111 /$ jse. 12229

Presl C. B. 1843. Hymenophyllaceae. Eine botanische Abhandlung. Prague, Haase, 70 pp.

Serizawa K. 1975. Pteridophytes of the Ryukyu Islands (1). Sci. Rep. Takao Mus. N. H. 7: 1-51.

Singh S., Panigrahi G. 2005. Ferns and Fern-allies of [Triap District] Arunachal Pradesh 1. Bishen Singh Mahendra Pal Singh, Dehra Dun, 426 pp. 
Sledge W. A. 1968. The Hymenophyllaceae of Ceylon. J. Linn. Soc. Bot. 60(383): 289-308. DOI: 10.1111/j.10958339.1968.tb00091.x

Tagawa M. 1959. Genshoku Nippon yoshi shokubutsu zukan. Coloured illustrations of the Japanese pteridophyta. Osaka, Hoikusha. 270 pp. [In Japanes].

Tagawa M., Iwatsuki K. 1979. Flora of Thailand. Vol. 3, part 1. Forest Herbarium, Royal Forest Department, Bangkok, 128 pp.

Tardieu-Blot, M., Christensen C. 1939. Hymenophyllacees. In: Flore generale de l'Indo-Chine. Ed. H. Lecomte. Massion Et. Cie, Paris, 51-72 pp.

Wu Y. D., Nwe T. Y., Sun J. Q., Zhang X. C. 2016. Additions to the Pteridophyte Flora of Xizang (V) Didymoglossum (Hymenophyllaceae), an unrecorded fern genus from Xizang, China. Newsletter of Himalayan Botany 50: 4-6.

Yoroi R., Iwatsuki K. 1977. An observation on the variation of Trichomanes minutum and allied species. Acta Phytotax. Geobot. 28: 152-159.

Zhang X. C. 2008. Miscellaneous Notes on Pteridophytes from China and neighbouring regions. In: Perspectives in Pteridophytes. Eds. S. C. Verma, S. P. Khullar, H. K. Cheema. Bishen Singh Mehendra Pal Singh, Dehradun, India, 29-33 pp. 